\title{
Evaluation of environmental factors effect on the genetic diversity, genetic structure and the potential distribution of Rhododendron aureum Georgi under changing climate
}

\author{
Wei Zhao ${ }^{1}$, Xiaolong Wang ${ }^{2}$, Lin $\mathrm{Li}^{3}$, Jiangnan $\mathrm{Li}^{1}$, Hang Ying ${ }^{4}$, Ying Zhao ${ }^{4}$, and Xia \\ Chen $^{1}$ \\ ${ }^{1}$ Jilin University \\ ${ }^{2}$ Qiqihaer Medical University \\ ${ }^{3}$ Qiqihar Medical University \\ ${ }^{4}$ Affiliation not available
}

October 8, 2020

\begin{abstract}
Understanding genetic variation and structure, adaptive genetic variation and its relationship with environmental factors is of great significance to understand how plants adapt to climate change and design effective conservation and management strategies. The objective of this study was to (I) investigate the genetic diversity and structure by AFLP markers in 36 populations of R. aureum from northeast China, (II) reveal the relative contribution of geographical and environmental impacts on the distribution and genetic differentiation of R. aureum; (III) identify outlier loci under selection and evaluate the association between outlier loci and environmental factors and (IV) exactly calculate development trend of population of R. aureum,as it is confronted with severe climate change and to provide information for designing effective conservation and management strategies. We found high genetic variation $(\mathrm{I}=0.584)$ and differentiation among populations $(\Phi S T=0.711)$ and moderate levels of genetic diversity within populations of R. aureum. A significant relationship between genetic distance and environmental distance was identified, which suggested that the differentiation of different populations was the caused by environmental factors. Using BayeScan and Dfdist, 42 outlier loci identified and most of the outlier loci are associated with climate or relief factors, suggesting that these loci are linked to genes that are involved in the adaptability of R. aureum to environment. Species distribution models (SDM) showed that climate warming will cause a significant reduction of suitable area for R. aureum especially under the RCP 85 scenario. Our results help to understand the potential response of $\mathrm{R}$. auruem to climatic changes, and provide new perspectives for $\mathrm{R}$. auruem resource management and conservation strategies.
\end{abstract}

Title:Evaluation of environmental factors effect on the genetic diversity, genetic structure and the potential distribution ofRhododendron aureum Georgi under changing climate

Authors:Wei Zhao ${ }^{1,2}$, Xiaolong $\mathrm{Wang}^{3}$, Lin $\mathrm{Li}^{3}$,Jiangnan $\mathrm{Li}^{1,2}$ Hang $\mathrm{Yin}^{4,5}$,Ying Zhao ${ }^{4,5}$ and Xia Chen ${ }^{1,2 *}$

${ }^{1}$ National \& Local United Engineering Laboratory for Chinese Herbal Medicine Breeding and Cultivation, Jilin University;

${ }^{2}$ School of Life Science, Jilin University;

${ }^{3}$ Medical technology department, Qiqihar Medical University;

4 Jilin Provincial Joint Key Laboratory of Changbai Mountain Biocoenosis and Biodiversity;

${ }^{5}$ Academy of Sciences of Changbai Mountain; 
* Correspondence: cbs1981@163.com, chenxia@163.com;

Abstract:Understanding genetic variation and structure, adaptive genetic variation and its relationship with environmental factors is of great significance to understand how plants adapt to climate change and design effective conservation and management strategies. The objective of this study was to (I) investigate the genetic diversity and structure by AFLP markers in 36 populations of $R$. aureum from northeast China, (II) reveal the relative contribution of geographical and environmental impacts on the distribution and genetic differentiation of $R$. aureum ; (III) identify outlier loci under selection and evaluate the association between outlier loci and environmental factors and (IV) exactly calculate development trend of population of $R$. aureum, as it is confronted with severe climate change and to provide information for designing effective conservation and management strategies. We found high genetic variation $(I=0.584)$ and differentiation among populations $(\Phi S T=0.711)$ and moderate levels of genetic diversity within populations of $R$. aureum - A significant relationship between genetic distance and environmental distance was identified, which suggested that the differentiation of different populations was the caused by environmental factors. Using BayeScan and Dfdist, 42 outlier loci identified and most of the outlier loci are associated with climate or relief factors, suggesting that these loci are linked to genes that are involved in the adaptability of $R$. aureum to environment. Species distribution models (SDM) showed that climate warming will cause a significant reduction of suitable area for $R$. aureum especially under the RCP 85 scenario. Our results help to understand the potential response of $R$. auruem to climatic changes, and provide new perspectives for $R$. auruemresource management and conservation strategies.

Keywords: Environmental factors, Rhododendron aureumGeorgi, Genetic diversity, Genetic structure, Distribution, Climate change

\section{Introduction}

Genetic diversity is the basic requirement for species to long-term survive and adapt to environmental changes on an evolutionary time scale(E.E.K. Donald A. Falk, 2001; Frankham, 2005). Genetic structure is important as it can provide insights into the history of a population, and the current levels and distribution of genetic variation can influence the future success of populations(Erickson, Hamrick, and Kochert (2004). Under any combination of natural selection and random genetic drift, populations separated by geographic distance may diverge due to reduced gene flow and population connectivity (isolation by geographical distance, IBD)(Nosil \& Rundle, 2012). Population divergence may still occur when reproductive isolation evolves between neighboring populations as a result of ecologically-based divergent selection in different environments (isolation by environment IBE)(I. J. Wang \& Bradburd, 2014). Geographical processes may influence the population genetic structure at large spatial scales, while ecological processes may influence the population genetic structure at small spatial scales(Sacks, Brown, \& Ernest, 2004).

Global climate change has become one of the major threats to biodiversity (M. B. Davis \& Shaw, 2001; Camille Parmesan, 2006). Species may respond to global climate change by local adaptation(Margaret B. Davis, Shaw, \& Etterson, 2008; C Parmesan, 2006), individual migration (Breshears, Huxman, Adams, Zou, \& Davison, 2008; Lenoir, Gegout, Marquet, de Ruffray, \& Brisse, 2008), range reduction(Thuiller, Lavorel, Araujo, Sykes, \& Prentice, 2005) or a combination of these(Margaret B. Davis et al., 2008). Local adaptation has been found to be a conventional way of responding to climate change in various plant species. (Coop, Witonsky, Di Rienzo, \& Pritchard, 2010; Gonzalez-Martinez, Krutovsky, \& Neale, 2006; Hancock et al., 2011; Savolainen, Pyhäjärvi, \& Knürr, 2007). Uncovering the genetic basis of local adaptations governed by natural selection is particularly important for understanding how plants adapt to their environment and respond to climate change. Reciprocal transplant experiments, quantitative trait locus (QTL) mapping and multiple-marker-based "neutrality" tests were used to investigate the local adaptations (Chartier, Pélozuelo, Buatois, Bessière, \& Gibernau, 2013; Storz, 2005; Tanksley, 1993). However, because reciprocal transplant experiments and QTL mapping need to be based on phenotypic variation as a starting point, these approaches are generally restricted to a consideration of measurable traits that have already been implicated as candidates for different selection by independent lines of evidence, and they are unsuited to analyse adaptive genetic responses to climate change for the species which experience long juvenile phase in their life 
history(Savolainen et al., 2007; Storz, 2005). Genome scans have been an approach to identify marker loci that are linked to selectively relevant target loci (outlier loci) through "genetic hitchhiking"(Luikart, England, Tallmon, Jordan, \& Taberlet, 2003), and are widely used to detect the local adaptation of species to environmental conditions(Magdy, Werner, McDaniel, Goffinet, \& Ros, 2016; T. Wang, Wang, Xia, \& Su, 2016; A. H. Yang, Wei, Fritsch, \& Yao, 2016b).Dfdist and BayeScan are two most commonly used methods. Dfdist builds an expected neutral distribution of $F_{S T}$ values under a classic symmetrical island model and loci potentially under positive selection can be identified if they exhibit unusually high $F_{S T}$ deviations from neutral estimates(M. A. Beaumont \& Balding, 2004; Mark A. Beaumont \& Nichols, 1996); BayeScan evaluates population-specific $F_{S T}$ values by considering different demographic histories and different amounts of genetic drift between populations(Foll \& Gaggiotti, 2008). In this method, $F_{S T}$-based population genomic methods can be used to seek adaptive loci by scanning a lots of markers such as amplified fragment length polymorphism (AFLP) technique (Bensch \& Akesson, 2005). The AFLP technique(Pieter Vos, 1995) has been commonly used to detect genetic diversity within and among populations, particularly in non-model organisms for which no prior genomic information is available. AFLP genome scans have been extensively employed to study plant populations, such as Liriodendron chinense (A. H. Yang, Wei, Fritsch, \& Yao, 2016a), Gentiana nivali s(Bothwell et al., 2013), Arabidopsis halleri (Meyer, Vitalis, Saumitou-Laprade, \& Castric, 2009), and Sphaeralcea ambigua (Shryock et al., 2015).

A major problem with genome scans is that they often detect false positives due to deviations from HardyWeinberg equilibrium and the assumption of the population structure model(L. Excoffier, Hofer, \& Foll, 2009). Natural selection along environmental gradient or heterogeneity generates gradual changes (i.e. clinal variation) in allele frequencies at loci linked to selected genes(Manel, Poncet, Legendre, Gugerli, \& Holderegger, 2010b). Consequently, outlier loci can potentially be detected by a closely association between allele frequencies and environmental parameters(Coop et al., 2010). The correlative approach need not consider the population structure and can be used to seek affirmation of outlier loci from the identification of candidate loci with genome scan methods(Joost et al., 2007; Nunes, Beaumont, Butlin, \& Paulo, 2011; T. Wang et al., 2016; A. H. Yang et al., 2016b).

Natural population responses to global climate change by changing their geographical distribution, and species distribution models (SDM) have become increasingly popular tools for predicting the geographic ranges of species and have been important for predicting changes in distribution from past or future climatic events and for conservation(Hijmans \& Graham, 2006; Kremen et al., 2008). Maxent, one of the most commonly used methods for inferring species distributions and environmental tolerances from occurrence data, allows users to fit models of arbitrary complexity(Warren \& Seifert, 2011). Maxent calculates probability distributions based on incomplete information and does not require absence data, making it appropriate for modeling species distributions based on presence-only herbarium records(Merow, Smith, \& Silander, 2013; Phillips SJ, 2006). During the past decades, many species' distribution have been studied by the Maxent, such as predicting habitat suitability of alien invasive weeds(Wan, Wang, Tan, \& Yu, 2017), predicting the potential distribution of threatened medicinal plants Fritillaria cirrhosa and Liliumnepalense(Rana, Rana, Ghimire, Shrestha, \& Ranjitkar, 2017), hindcasting the distributions of neotropical savanna tree species during the Last Glacial Maximum and Last Inter-Glacial(Bueno et al., 2017).

Rhododendron aureum Georgi (syn. Rh. Chrysanthum Pall.), the target plant species in this study, is a perennial evergreen creeping shrub with a large number of branched stems inhabiting alpine regions of Korea, China, Japan, and the Kamchatka peninsula. This plant can grow up to $1 \mathrm{~m}$ in height, and blooms from June to July in Korea with pale yellow flowers. It has been shown to always occupy the snowmelt gradient and especially to dominate in early exposed places(Kudo, 1992). In China, it grows mainly in the alpine tundra and the Betula ermaniipopulation belts of Changbai Mountain, ranging from 1,000 to 2,506 m a.s.l.(Kudo, 1993). The $R$. aureum is one of the constructive and dominant species on the alpine tundra ecosystem, and it plays an important role in maintaining the ecological balance and preventing and controlling soil erosion.

Alpine environment is locally variable as small changes in altitude can lead to large changes in temperature, humidity, exposure, and other types of changes(Byars, Papst, \& Hoffmann, 2007; Hovenden \& Jkvander, 
2004). With the global climate changing, in some alpine area, the increase in air temperature was more than twice as great as the increase in global mean air temperature during the 20th century(Bohm et al., 2001). Plant species are particularly vulnerable under the climate changing environment in alpine. Understanding the contemporary and historical ecological (climatic, geographical) factors shaping population genetic diversity is of great significance for studying molecular ecology, conservation biology and evolutionary biology (And \& Hamrick, 1984; Holderegger, Buehler, Gugerli, \& Manel, 2010).

In this study we adopted AFLP markers for characterizing the adaptive loci under selection using BayeScan and Dfdist, employed Multiple Linear Regression (MLR) to detect potential adaptive loci that are under selection from existing environmental factors, and using species distribution models (SDM) to predict potential distribution of $R$. aureum during the Last Glacial Maximum (LGM) and the future. The objective of this study was to (i) investigate the genetic variation and genetic structure of $R$. aureum; (ii) reveal the relative contribution of geographical and environmental impacts on the distribution and genetic differentiation of $R$. aureum ; (iii) identify outlier loci under selection and assess the association between outlier loci and climate and (iv) exactly calculate development trend of population of $R$. aureum , as it is confronted with severe climate change and to provide information for designing effective conservation and management strategies.

2. Materials and Methods

\subsection{Study site and plant material}

Changbai Mountain is generally recognized as the highest peak in northeast China and eastern Eurasia, with obvious mountain climate characteristics. This environment is locally variable as small changes in altitude can lead to large changes in temperature, humidity, exposure, and other types of changes(X. Yang \& Wu, 1998). The alpine region on Changbai Mountain is the southern boundary of alpine tundra in east Eurasia. The varied topography, weather, soil and other natural conditions have created rich biodiversity and vertical zonal distribution of vegetation on Changbai Mountain, which has more than 2,277 species of plants and a notable richness of endemic species.

From 2012 to 2014, fresh leaves were collected from 461 individuals belong to 36 nature populations of $R$. aureum along an environmental transect which altitude range from $1200 \mathrm{~m}$ to $2600 \mathrm{~m}$ a.s.l., annual mean temperature range from $0.1 \mathrm{degC}$ to $-6.6 \mathrm{deg} \mathrm{C}$ and annual precipitation range from $761 \mathrm{~mm}$ to $1096 \mathrm{~mm}$ (Table 1, Figure C). These populations were scattered along peaks of Changbai Mountain (32 populations), Laobai Mountain (2 populations), and Wangtian'e Mountain (2 populations)(Figure 1) . In each of the population, a random sample from 7 to 17 individuals was obtained. In each locality, individual samples were taken from plants separated by at least $5 \mathrm{~m}$ (in order to avoid sampling clones) and dried directly in silica gel for transport back to Jilin University for subsequent DNA extraction.

Table 1. Summary of AFLP variation for 36 populations of

R. aureum.

\begin{tabular}{|c|c|c|c|c|c|c|c|}
\hline Pop & $\begin{array}{l}\text { GPS } \\
\text { Coordinates }(\mathrm{N}\end{array}$ & $\begin{array}{l}\text { GPS } \\
\text { /E } \phi \text { ordinat }\end{array}$ & /ADtitude (m) & $\mathrm{N}$ & PPL & I & $\mathrm{H}$ \\
\hline N1 & 42.0292 & 128.0664 & 2600 & 12 & 41.871 & 0.228 & 0.154 \\
\hline $\mathrm{N} 2$ & 42.04018 & 128.0678 & 2300 & 8 & 58.797 & 0.329 & 0.224 \\
\hline N3 & 42.04202 & 128.0686 & 2200 & 14 & 31.403 & 0.153 & 0.100 \\
\hline N4 & 42.04575 & 128.0706 & 2100 & 14 & 22.272 & 0.123 & 0.083 \\
\hline N5 & 42.05552 & 128.0699 & 2000 & 10 & 37.862 & 0.208 & 0.142 \\
\hline N6 & 42.05935 & 128.063 & 1800 & 11 & 33.185 & 0.188 & 0.128 \\
\hline N7 & 42.09014 & 128.0687 & 1580 & 14 & 2.450 & 0.014 & 0.010 \\
\hline N8 & 42.13372 & 128.191 & 1200 & 14 & 18.708 & 0.103 & 0.070 \\
\hline NW1 & 41.99727 & 128.025 & 2462 & 14 & 52.116 & 0.293 & 0.200 \\
\hline NW2 & 42.0021 & 128.0249 & 2590 & 14 & 56.570 & 0.315 & 0.214 \\
\hline NW3 & 42.01297 & 128.0212 & 2161 & 9 & 30.958 & 0.166 & 0.112 \\
\hline
\end{tabular}




\begin{tabular}{llllllll}
\hline & GPS & \multicolumn{2}{l}{ GPS } & & & & \\
Pop & Coordinates(N/E E ordinates(N/R)titude $(\mathrm{m})$ & $\mathrm{N}$ & $\mathrm{PPL}$ & $\mathrm{I}$ & $\mathrm{H}$ \\
\hline NW4 & 42.0164 & 128.0252 & 2501 & 14 & 33.185 & 0.179 & 0.121 \\
NW5 & 42.02153 & 128.0425 & 2198 & 7 & 32.071 & 0.176 & 0.119 \\
NW6 & 42.02443 & 128.0402 & 2341 & 10 & 55.011 & 0.302 & 0.205 \\
NW7 & 42.03347 & 128.0433 & 2431 & 14 & 41.425 & 0.212 & 0.140 \\
NW8 & 42.03802 & 128.0485 & 2315 & 14 & 38.307 & 0.193 & 0.128 \\
NW9 & 42.02957 & 128.0385 & 2500 & 7 & 39.421 & 0.225 & 0.153 \\
NW10 & 42.043 & 128.0515 & 2122 & 14 & 36.526 & 0.189 & 0.126 \\
W1 & 41.99007 & 128.0001 & 2223 & 14 & 35.857 & 0.196 & 0.133 \\
W2 & 41.98912 & 128.0119 & 2138 & 14 & 32.517 & 0.163 & 0.109 \\
W3 & 41.98713 & 128.0049 & 2014 & 14 & 30.067 & 0.154 & 0.102 \\
W4 & 41.97795 & 128.0006 & 1872 & 14 & 33.408 & 0.171 & 0.114 \\
S1 & 41.97879 & 128.0636 & 2535 & 14 & 48.998 & 0.257 & 0.172 \\
S2 & 41.95675 & 128.0826 & 2099 & 14 & 34.744 & 0.179 & 0.12 \\
S3 & 41.95501 & 128.0794 & 2083 & 14 & 37.639 & 0.198 & 0.133 \\
S4 & 41.95376 & 128.0762 & 2030 & 14 & 50.78 & 0.278 & 0.188 \\
S5 & 41.95178 & 128.0752 & 1945 & 14 & 35.412 & 0.177 & 0.118 \\
S6 & 41.94115 & 128.0488 & 1814 & 14 & 30.735 & 0.181 & 0.125 \\
NE1 & 42.0542 & 128.0714 & 2000 & $\mathrm{~T}$ & 33.63 & 0.189 & 0.129 \\
NE2 & 42.04927 & 128.0738 & 2100 & 9 & 29.176 & 0.172 & 0.118 \\
NE3 & 42.04517 & 128.0749 & 2200 & 14 & 29.844 & 0.159 & 0.107 \\
NE4 & 42.04015 & 128.0681 & 2300 & 14 & 29.399 & 0.156 & 0.105 \\
L1 & 44.10465 & 128.0431 & 1698 & 14 & 39.866 & 0.218 & 0.147 \\
L2 & 44.10325 & 128.0426 & 1701 & 14 & 34.967 & 0.185 & 0.125 \\
WTE1 & 41.72863 & 127.902 & 2048 & 14 & 25.612 & 0.124 & 0.083 \\
WTE2 & 41.72845 & 127.9011 & 2044 & 14 & 26.949 & 0.115 & 0.074 \\
Total & - & - & - & 461 & 99.777 & 0.584 & 0.402 \\
\hline
\end{tabular}

N, population size; PPL, The percentage of polymorphic loci; H, Nei's (1973) gene diversity; I, Shannon's Information index
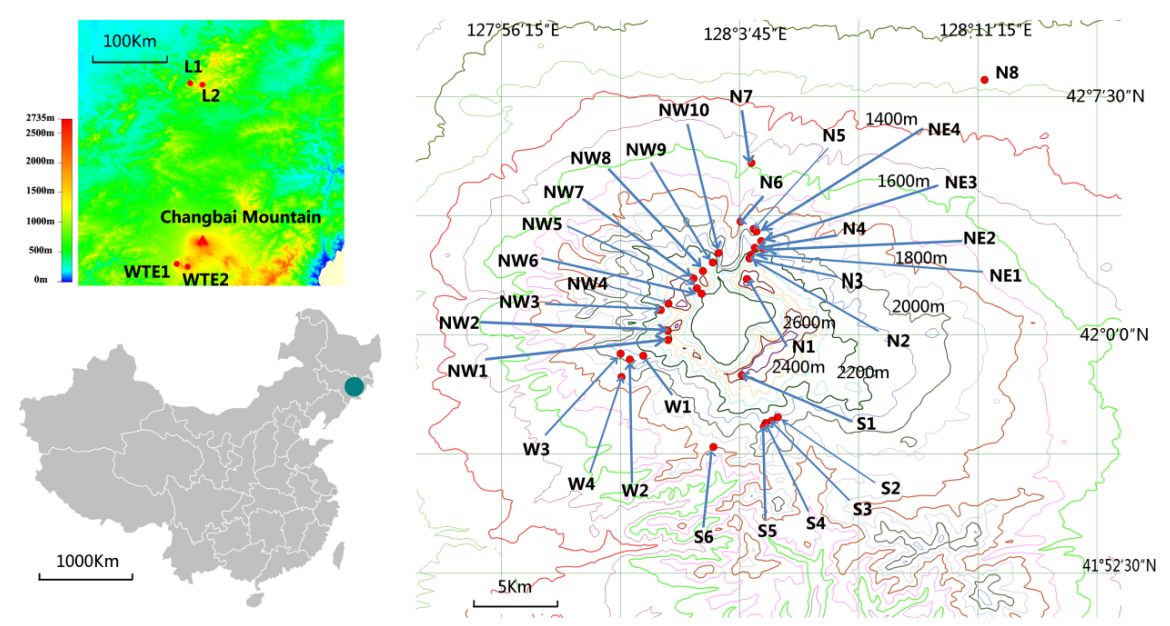

Figure 1. Distribution of $36 R$. aureum populationssampled from China. We extracted the elevational information from DIVA-GIS Free Spatial Data (http://www.diva-gis.org/Data) and created the map by the 
program Global Mapper version 13.00 (http://www.bluemarblegeo.com/products/global-mapper.php) and DIVA-GIS version 7.5.0.

\subsection{DNA extraction, AFLP and marker scoring}

The total DNA was extracted from the dried leaves by using a Plant Genomic DNA Kit (Bioteke Beijing Co. Ltd., Beijing, China). The DNA samples were diluted to $10 \mathrm{ng} / \mu \mathrm{l}$ and stored at $-20^{\circ} \mathrm{C}$ until further analysis. AFLP marker was carried out according to the method of Vos et al. [30] with the following little modifications: the digestion-ligation reaction was performed in a $10 \mu$ l containing $1 \mu l$ of $10 \times \mathrm{T} 4$ ligation buffer, $0.2 \mathrm{mM}$ of ATP, $50 \mathrm{ng}$ DNA, $1 \mathrm{U}$ of T4 DNA ligase (Fermentas, Shenzhen, China), $1 \mathrm{U}$ of Eco RI (Fermentas, Shenzhen, China), 1 U of Mse I (New England Biolabs, Beijing LTD), $1.0 \mu \mathrm{M}$ ofMseI -Adapter and $0.1 \mu \mathrm{M}$ of Eco RI-Adapter and double-distilled water. The reaction was incubated at $37{ }^{\circ} \mathrm{C}$ for $8 \mathrm{~h}$, $16{ }^{\circ} \mathrm{C}$ for $4 \mathrm{~h}$, inactivated at $65{ }^{\circ} \mathrm{C}$ for $10 \mathrm{~min}$, and stored at $4{ }^{\circ} \mathrm{C}$. Pre-amplification was performed in a $25 \mu \mathrm{l}$ containing $2.5 \mu \mathrm{l}$ of $10 \times$ PCR buffer and $0.5 \mathrm{U}$ of Taq polymerase(Transgen Biotech Beijing Co. Ltd., China ), $2.5 \mu \mathrm{l}$ of diluted digestion-ligation product, $0.2 \mu \mathrm{M}$ of dNTPs, $0.3 \mu \mathrm{M}$ each of primers with a single selective nucleotide. The pre-amplification conditions was as follows: pre-denatured at $94{ }^{\circ} \mathrm{C}$ for 5 min and 30 cycles of $94{ }^{\circ} \mathrm{C}$ for $30 \mathrm{~s}, 56{ }^{\circ} \mathrm{C}$ for $60 \mathrm{~s}$, and $72{ }^{\circ} \mathrm{C}$ for $80 \mathrm{~s}$, with a final extension for $10 \mathrm{~min}$ at $72{ }^{\circ} \mathrm{C}$. The pre-amplification products were diluted 1 to $40(\mathrm{v} / \mathrm{v})$ with $\mathrm{ddH}_{2} \mathrm{O}$.

The selective amplification was essentially the same as that for pre-amplification except that $2 \mu \mathrm{L}$ diluted pre-amplification product was used as template, and $2 \mu \mathrm{M}$ Eco RI and Mse I selective primer were used. 10 pairs of primers were selected for selective amplification (Table A ). The selective amplification reaction had two cycle sets: pre-denatured at $94{ }^{\circ} \mathrm{C}$ for 5 min, 13 cycles of $94{ }^{\circ} \mathrm{C}$ for $30 \mathrm{~s}, 65{ }^{\circ} \mathrm{C}$ (which was lowered $0.7{ }^{\circ} \mathrm{C}$ at each cycle) for $30 \mathrm{~s}$ and $72{ }^{\circ} \mathrm{C}$ for $60 \mathrm{~s}$, followed by 18 cycles of $94{ }^{\circ} \mathrm{C}$ for $30 \mathrm{~s}, 56{ }^{\circ} \mathrm{C}$ for $30 \mathrm{~s}$ and 72 ${ }^{\circ} \mathrm{C}$ for $80 \mathrm{~s}$. After selective amplification, the products were added $25 \mu \mathrm{l}$ of formamide loading buffer (98\% deionized formamide, $10 \mathrm{mM}$ EDTA, $0.1 \%$ bromophenol blue, and $0.1 \%$ xylene cyanol). Then the products were denatured at $95{ }^{\circ} \mathrm{C}$ for 5 min and quickly cooled on ice, and separated on $6 \%$ denaturing polyacrylamide gel in $1 \times$ TBE buffer at $70 \mathrm{~W}$ for $4.5 \mathrm{~h}$. Gels were stained according to the silver staining method(Bassam BJ, 1991).

\subsection{Data analysis}

\subsubsection{Genetic Diversity and Genetic Structure}

The AFLP bands were scored as present (1) or absent (0), and the AFLP band data were transferred to a binary $(1 / 0)$ data matrix for further analysis. Shannon's information index $(I)(\mathrm{RC}, 1972)$, percentage of polymorphic loci $(P P L)$, and genetic distance were estimated using the POPGENE v1.31 (Yeh F, 1997). Total genetic diversity $\left(H_{T}\right)$ and mean genetic diversity within populations $\left(H_{S}\right)$ were calculated using Nei's(Nei, 1973) genetic diversity statistics. A UPGMA tree based on genetic distance(Nei, 1978) was performed among different populations to identify their genetic relationships using NTsyspc v2.02 (Rohlf, 1997).

Population genetic structure was further assessed using model-based Bayesian assignment as implemented in STRUCTURE 2.3.4 software(Pritchard, Stephens, \& Donnelly, 2000). Clustering of individuals was constructed without using the geographical origin of the samples as an informative prior. Analyses were based on an admixture ancestry model with correlated allele frequencies for a range of $\mathrm{K}$ genetic clusters from 1 to 36, with 20 replicates per K. The analyses were performed with a burn-in period and a run length of the Monte Carlo Markov chain (MCMC), of 10000 and 100000 iterations, respectively. The most likely number of genetic clusters $(\mathrm{K})$ was estimated by the $\Delta \mathrm{K}$ statistic (Evanno, Regnaut, \& Goudet, 2005), using Structure Harvester (Earl \& Vonholdt, 2012). Then CLUMPP 1.1.2(Jakobsson \& Rosenberg, 2007) was used to align the 20 runs of the most representative $\mathrm{K}$ value and to compute the pairwise symmetric similarity coefficients $(G)$ between pairs of runs, the average pairwise similarity $(H)$ for the 20 replicates and the average probability of belonging to each cluster $(\mathrm{Q})$. For $\mathrm{K}=2$, the full search method with 1000 replicates was used. A hierarchical analysis of molecular variance (AMOVA) was used to determine genetic differentiation $\left(F_{S T}\right)$ within and among the groups. A nested AMOVA taking into consideration the two main genetic groups resulting from the Bayesian clustering with STRUCTURE $(\mathrm{K}=2)$. using ARLEQUIN 
version 3.5.1.2(L Excoffier \& Lischer, 2010).

\subsubsection{Outlier Detection}

Two complementary methods were used to detect outlier loci of all populations of $R$. aureum. BayeScan version 2.1. identifies markers with unusually high or low levels of genetic differentiation as outliers that have signatures of diversifying or balancing selection, respectively (Foll \& Gaggiotti, 2008). Specifically, selection is concluded from an AFLP marker if the marker-specific estimates of $F_{S T}$ are needed in addition to population-specific estimates to interpret observed patterns of differentiation in the dataset(Fischer, Foll, Excoffier, \& Heckel, 2011). A threshold value for determining loci under selection was estimated according to Jeffreys' interpretation(Harris, 1961), i.e., $\log _{10} \mathrm{PO}>2.0$ was regarded as adequate evidence for selection. A threshold of $\log 10 \mathrm{PO}>2.0$ was employed to reject the null hypothesis in each of the conducted tests. The analysis was performed with 20 pilot runs and a 50,000 step burn-in followed by 50,000 iterations and a thinning interval of 10 for the set of polymorphic AFLP markers. The false discovery rate (FDR ) calculated the expected proportion of false positives for statistically significant results(Foll \& Gaggiotti, 2008). We used the software Dfdist to simulate a null distribution of $F_{S T}$ values under an island model, which was relative insensitive to demographic structure, population structure and mutation level. Simulations were conducted with a mean $F_{S T}$ equal to the trimmed mean $F_{S T}$, which was calculated by excluding $30 \%$ of the most extreme $F_{S T}$ values observed in the empirical dataset. We analysed the distributions of the $F_{S T}$ values over all loci to null hypothesis of neutral evolution. Loci with a high or low $F_{S T}$ value were taken as potentially under selection. In the study, we simulated the neutral distribution of $F_{S T}$ with 10,000 iterations at the $99.9 \%$ confidence intervals.

\subsubsection{Environmental data and correlation analyses}

To characterize environmental differences, BIOCLIM variables were obtained for each of the 36 sites by extrapolating climate data to the GPS coordinates for each population using DIVA-GIS software (Hijmans, Cameron, Parra, Jones, \& Jarvis, 2005; Hijmans, Guarino, Cruz, \& Rojas, 2001). The BIOCLIM dataset includes 19 variables that describe monthly temperature and precipitation patterns for a spatial resolution of $1 \mathrm{~km}^{2}$ (http://www.worldclim.org/). The sampled area spanned all the known $R$. aureum range in China, which occurs primarily in the alpine, along an annual mean precipitation gradient from 761-1096 $\mathrm{mm}$ and annual mean temperature gradient from $-6.6^{\circ} \mathrm{C}$ to $0.1^{\circ} \mathrm{C}$. Elevation, slope, and topographic index were derived from a $90 \mathrm{~m}$ digital elevation model. The data set is provided by International Scientific \& Technical Data Mirror Site, Computer Network Information Center, Chinese Academy of Sciences (http://www.gscloud.cn). A principal component analysis (PCA) was applied to these environmental variables to examine possible correlations between eco-climatic variables and elevation and remove redundant variables (i.e. variables that were correlated at $|\mathrm{r}|>0.8$ and which were logically related). We first identified variables correlated to each retained axis, creating groups of variables. Within each group, we kept only one (or two) variables considered to be the most pertinent in terms of local adaptation in plants. Finally, eleven factors (Bio 1, annual mean temperature; Bio 2, mean diurnal range; Bio 3, Isothermality; Bio 4, temperature seasonality; Bio 5, max temperature of warmest month; Bio 9, mean temperature of driest quarter; Bio 16, precipitation of wettest quarter; Bio 17, precipitation of driest quarter; slp, slope; asp, aspect; tpi, topographic position index) were chosen as representative of environment factors.

Prior to subsequent analyses, environment data were $\log _{10}(x+1)$ transformed to improve normality and reduce heteroscedasticity. Dissimilarity matrices of Euclidean distances were calculated among normalized environment variables using $\mathrm{R}$ package. A matrix of geographic distances among sites was generated from GPS coordinates with the AFLP data in R software(Ehrich, 2006) and also $\log _{10}(\mathrm{x}+1)$ transformed. The genetic distance matrices of $R$. aureum was calculated with PopGene(Yeh F, 1997) using the Jaccard dissimilarity method of the scored bands, were tested against the Euclidean distance of the environment variables while controlling of the geographic distance matrix. The Mantel test was performed using the default preset values and parameters (999 permutation steps) according to the software manual using R program "vegan" package. Multiple matrix regression with randomization (MMRR) is a novel and robust approach for estimating the independent effects of potential factors, and the analysis was implemented with 10,000 permutations 
in $\mathrm{R}$ with the MMRR function script(Goslee \& Urban, 2007; I. J. Wang, 2013; Wu, Yu, Wang, Li, \& Xu, 2015).

Then, to detect relationships between allele frequencies and environmental variables, we applied Multiple Linear Regression (MLR)(Zulliger, Schnyder, \& Gugerli, 2013) in R v.3.3.3 to determine potential adaptive loci that are under selection from existing environmental factors. For outlier loci identified by both Dfdist and BayeScan, we estimated their population pairwise frequencies of AFLP alleles at the 36 sites. We selected values of the eleven selected environmental factors from each population. We then regressed the allele frequencies of the retained outlier loci (dependent variables) on the selected environmental variables (explanatory variables; standardized) using the MLR model. Potential adaptive loci were identified as $R_{\text {adj }}{ }^{2}>$ 0.5. Univariate regressions were then applied to each variable individually to estimate its significance.

\subsubsection{Species distribution model (SDM)}

We used Maxent 3.3.3k to predict distribution changes for $R$. aureum as a result of climate changing. Maxent is a program for maximum entropy modelling of the geographical distributions of species; it combines presence-only data with ecological-climatic layers to predict suitable areas (Phillips SJ, 2006; Phillips \& Dudik, 2008). For current distribution, we downscaled climate grids for the periods 1970-2000. In addition to sample locations in this study, we also collected the distribution records of $R$. aureum from the Chinese Virtual Herbarium (http://www.cvh.ac.cn/ ). After removing duplicate records, it remained a total of 42 records of $R$. aureum (Table B ) that were used to establish the distribution model by using 19 bio-climatic data layers from the WorldClim database. Most of the default parameters of Maxent were applied to conduct SDM, except the following user-selected parameters: application of random seed and random test percentage of $70 \%$, replicates of 10 and bootstrap as the replicated run type. The logistic output of Maxent consists of a grid map with each cell having an index of suitability between 0 and 1 . Low values show that the conditions are unsuitable for the species to live, whereas high values show that the conditions are more suitable. Model predictions were visualized using DIVA-GIS(Hijmans et al., 2001).

To obtain the distribution of $R$. aureum at the Last Glacial Maximum, we projected correlation between current species-climate and the LGM using the Community Climate System Model (CCSM4) scaled down to a 2.5-arcmin resolution. We used the Hadley Global Environment Model 2 (HadGEM2-ES) as a general circulation model under two climate scenarios (IPCC-CMIP5 RCP 26/85) to ensure the accuracy of assessment. The RCP 85 scenario represents a higher predicted greenhouse gas emission than RCP 26 .

3. Results

\subsection{Patterns of AFLP variation and population structure 3.1.1. Subsubsection}

The ten AFLP primer combinations generated 449 unambiguously scorable bands ranging from 1500 to 100 bp in 461 individuals from 36 natural populations. Of these fragments, $99.777 \%$ (448) were polymorphic. The overall Shannon's Information index $(I)$ and Nei's genetic diversity $(H)$ was 0.584 and 0.402 respectively. $R$. aureum showed high genetic diversity at the species level. Iranged from 0.014 to 0.329 and $H$ ranged from 0.01 to 0.228 within population (Table 1 ) . The genetic diversity of most populations is at moderate level; but there is still a low genetic diversity of some populations, such as population N7.

Bayesian clustering analyses performed with the software STRUCTURE indicated that the most informative representation of overall genetic structure was achieved for $K=2$, where $K$ is the number of subpopulations. The southern slope populations (populations S1-S6) and the western populations on the U-shaped valley of the northern slope (populations NW7 and NW8) formed cluster 2, while populations NW9 and NW10 showed a high degree of membership to cluster 2. Populations L1 and L2, which are not on the prominent peak of Changbai Mountain and populations from the western slope region showed that, with exception of populations NW3 and NW4, individuals had a high level of admixture. The remaining populations displayed a high degree of membership to cluster 1

(Figure 2). 

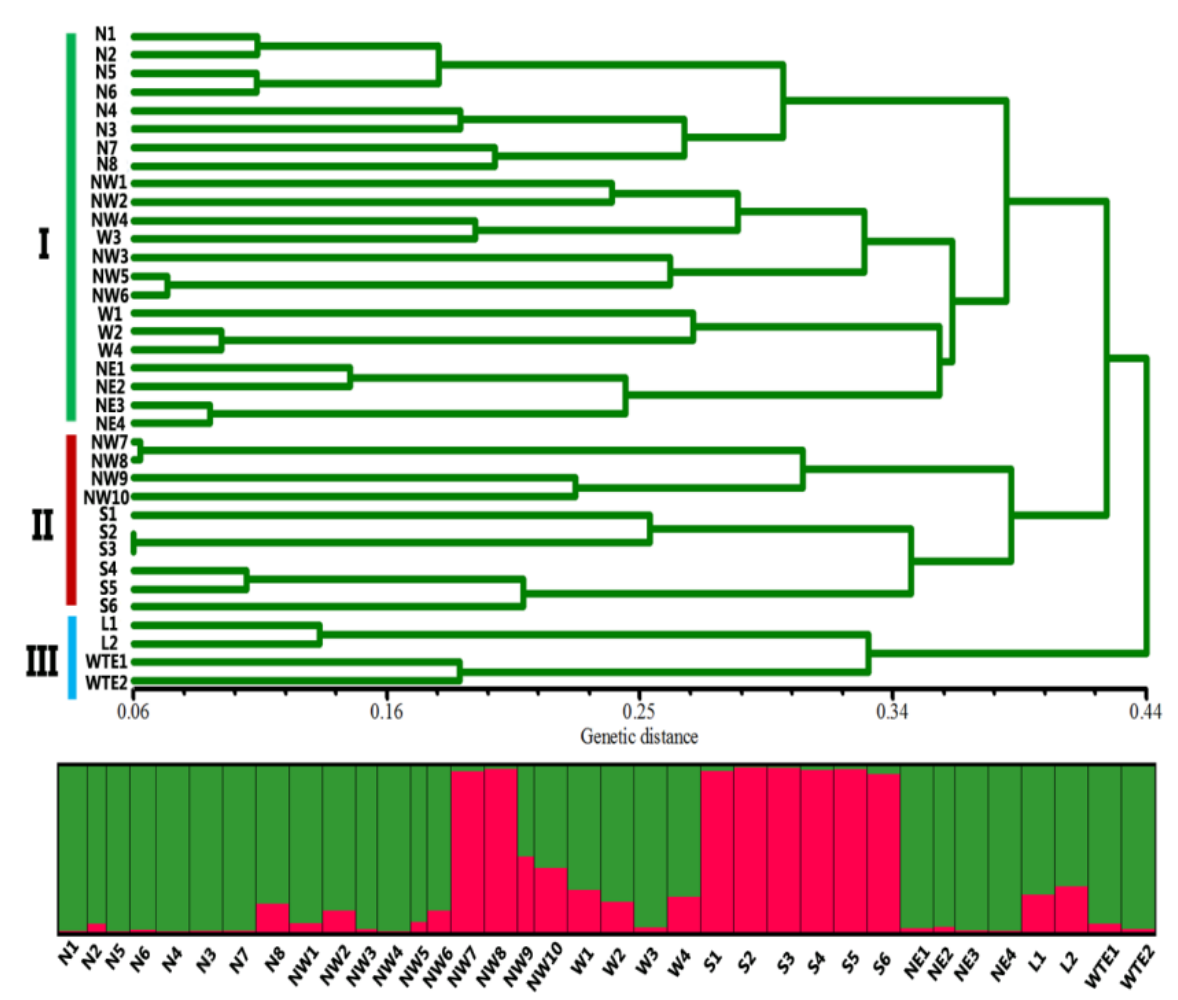

Figure 2. Phylogenetic relationships among 36 populations of $R$. aureum . inferred from AFLP data using a UPGMA tree (up) and with the STRUCTURE result at $\mathrm{K}=2$ (below).

The consensus UPGMA tree of populations contained three main groups (Figure 2). Group I contained all the northern, western, and northeastern slope populations. Group II was composed of all the populations from the southern slope and some populations on the western end of the U-shaped valley (populations NW7NW10). The remaining populations (populations L1, L2, WTE1, and WTE2), which do not occupy the peak of Changbai Mountain, clustered in group III.

An AMOVA (Table 2) attributed $68.87 \%$ of the overall genetic variation to the among-population component. A nested AMOVA that considered the two main genetic groups based on the Bayesian clustering analysis with the software STRUCTURE $(K=2)$ attributed $12.31 \%$ of the global variation to differences between the two clusters, 58 . $78 \%$ to among-populations within clusters and $28.91 \%$ to within populations $(\Phi \mathrm{ST}=0.711$; both at $P<0.0001)$.

Table 2. AMOVAs for AFLP variation surveyed in a total of 36 populations of

R. aureum.

Structure analyzed and source of variation d.f.

\section{(a) Global analysis}

Among populations

Within populations

(b) Grouping following STRUCTURE clustering ( $\mathrm{K}=2$ ) Among clusters

Among populations within clusters

Within populations (a) Global analysis

35

425

(b) Grouping following STRUCTURE clusterin 1

34

425 


\subsection{Correlations between genetic variation and environmental versus geographical factors}

The Mantel test showed a significant correlation between genetic distance and environmental distance $(r=$ $0.4871, P=0.001$ ), but between genetic distance and geographical distance was a non-significant correlation $(r=0.0971, P=0.028)$. When geographical factors were controlled, a partial Mantel test also showed isolation by environmental distance $(r=0.4797, P=0.001)$. Whereas environmental factors were controlled, we could not detect significant correlations between genetic differentiation and geographical distance $(r=$ $0.0118, P=0.378)$. The MMRR analysis indicated that the environment factors had large regression coefficient, whereas the effects of geographic distance were not significant (geographic distance: $\beta=0.0094$, $P=0.1075$; environment distance: $\beta=0.2372, P=0.0001$; Table 3 ).

Table 3. Results of the Mantel test, partial Mantel test and MMRR analyzing the correlation between geographical distances, environmental distances and Nei's genetic distance based on AFLP data.

\begin{tabular}{|c|c|c|c|c|c|c|}
\hline & Mantel test & Mantel test & Mantel test & partial Mantel test & partial Mantel test & partial Mantel test \\
\hline & $r$ & $P$ value & & & $r$ & $P$ value \\
\hline Gen.Geo & 0.0971 & 0.028 & 0.028 & 0.0118 & 0.0118 & 0.378 \\
\hline Gen.Env & 0.4871 & 0.001 & 0.001 & 0.4797 & 0.4797 & 0.001 \\
\hline
\end{tabular}

Regular letters refer to non-significant results and bold letters refer to significant correlations. Geo, geographical distance; Gen, genetic distance; Env, environmental distance.

\subsection{Outlier analyses and $M L R$ analysis}

BayeScan determined 71 loci as outliers with a $\log _{10} \mathrm{PO}$ above 2 , which is a threshold for adequate evidence for accepting a model under selection, corresponding to a posterior probability greater than 0.99 (Figure 3A ). Using the Dfdist, we identified 126 adaptive loci at the $99.5 \%$ confidence intervals (Figure 3B) . 42 outlier loci were identified using two complementary analyses. The extremely strict significance criteria in the two approaches also assured the robustness of 42 outlier loci. Lastly, 21 potential loci under selection were verified by the MLR analysis with $R_{a d j}{ }^{2}>0.5$ (Table 4 ). When we ran linear regressions using each environmental variable individually, all these eleven environmental variables were associated with the potential loci under selection and 30 loci were significantly $(\mathrm{P}<0.05)$ associated with at least one of the eleven selected environmental variables. 


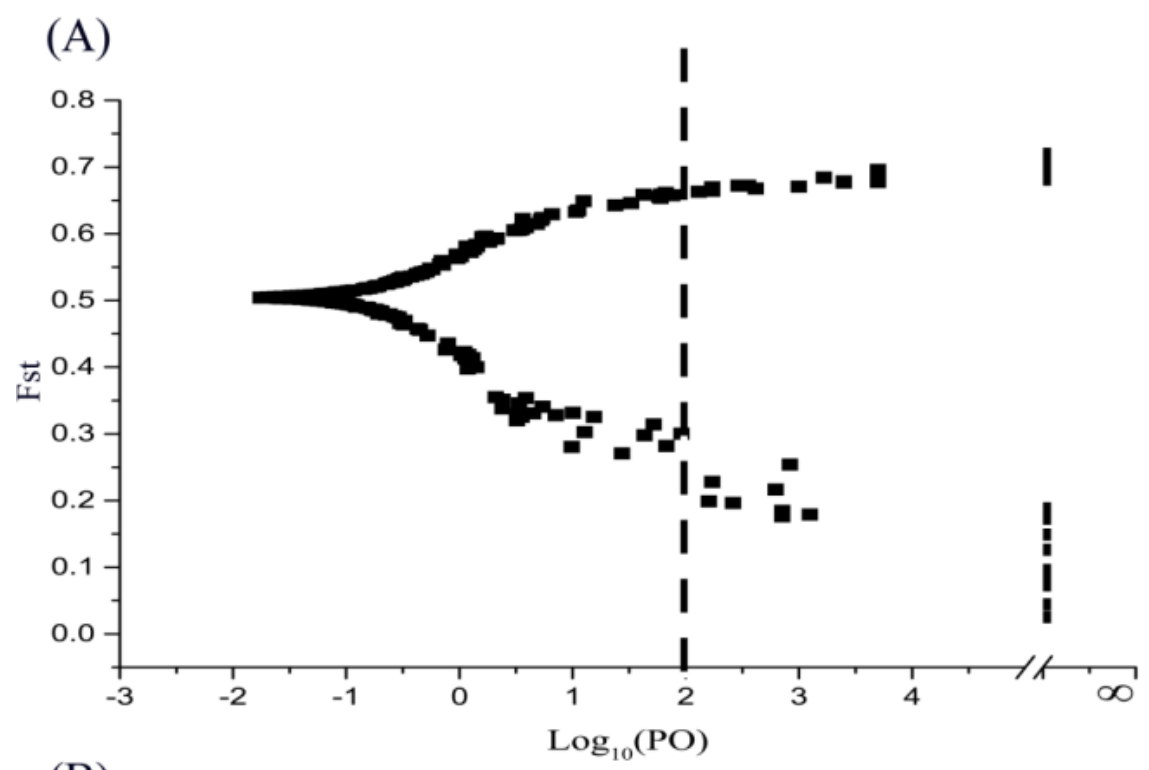

(B)

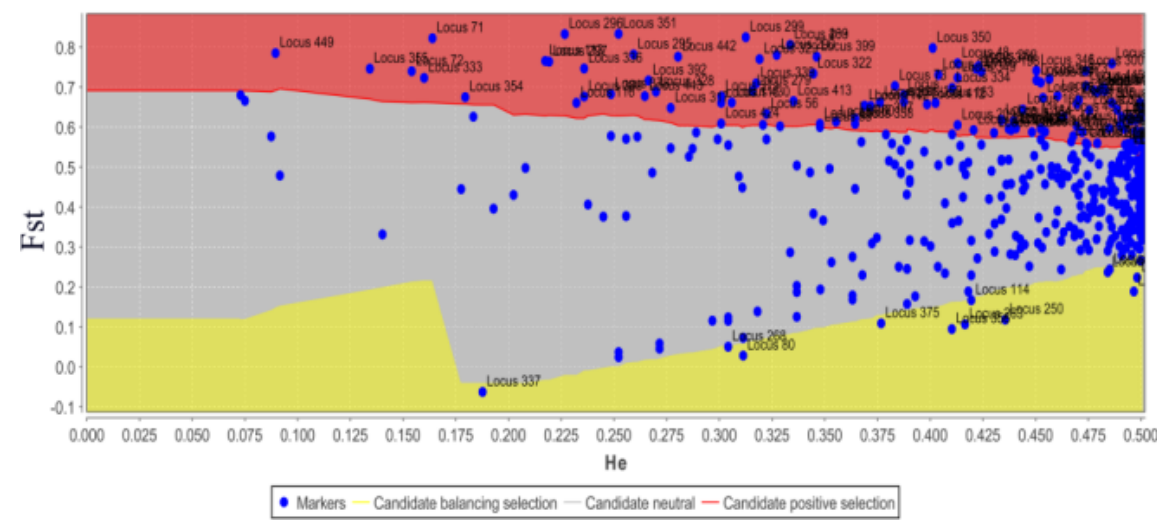

Figure 3. Outlier loci identified by BayeScan and Dfdist. (A ) Plot of $F_{S T}$ values and $\log _{10} \mathrm{PO}$ for 461 loci identified using BayeScan. Lines $\log _{10} \mathrm{PO}=2$ indicate "decisive" evidence for selection corresponding to a posterior probability of 0.99 . Solid black dots greater than $\log _{10} \mathrm{PO} 2$ represented outlier loci. (B) Outlier detection performed with Dfdist. Plot of $F_{S T}$ values of 461 loci in $R$. aureumpopulations was against heterozygosity.

Table 4. Results of outliers and environmental association analyses on AFLP loci of $R$. aureum

\begin{tabular}{lllll}
\hline Outlier ID & Environmental variables $\mathrm{R}_{\text {adj }}$ & Significant environmental variables & Outlier ID & Environmental variables R \\
\hline $\mathbf{L 4 8}$ & $\mathbf{0 . 5 2}$ & BIO3,BIO9 & L348 & 0.43 \\
L73 & $\mathbf{0 . 6 4}$ & BIO1, BIO3, BIO9 & L349 & 0.39 \\
L198 & 0.45 & BIO2, slp & L350 & $\mathbf{0 . 5 8}$ \\
$\mathbf{L 2 8 3}$ & $\mathbf{0 . 5 2}$ & BIO2 & L352 & $\mathbf{0 . 5 3}$ \\
$\mathbf{L 2 8 7}$ & $\mathbf{0 . 7 3}$ & BIO2, BIO16, BIO17, tpi & L373 & 0.30 \\
$\mathbf{L 2 8 9}$ & $\mathbf{0 . 6 1}$ & BIO1, BIO3 & L374 & 0.36 \\
$\mathbf{L 2 9 0}$ & $\mathbf{0 . 5 4}$ & BIO1, BIO3 & L399 & $\mathbf{0 . 6 4}$ \\
$\mathbf{L 2 9 1}$ & $\mathbf{0 . 5 0}$ & BIO1, BIO3 & L403 & $\mathbf{0 . 6 3}$ \\
$\mathbf{L 2 9 2}$ & $\mathbf{0 . 5 1}$ & BIO3 & $\mathbf{L 4 0 4}$ & $\mathbf{0 . 5 5}$
\end{tabular}




\begin{tabular}{lllll}
\hline Outlier ID & Environmental variables $\mathrm{R}_{\text {adj }}$ & Significant environmental variables & Outlier ID & Environmental variables R \\
\hline $\mathbf{L 2 9 3}$ & $\mathbf{0 . 6 6}$ & BIO3 & L405 & 0.49 \\
L294 & $\mathbf{0 . 5 7}$ & BIO3 & L445 & 0.28 \\
L298 & $\mathbf{0 . 5 8}$ & BIO3,tpi & L53 & 0.34 \\
L299 & $\mathbf{0 . 6 5}$ & BIO2, BIO3, tpi & L59 & 0.42 \\
L300 & 0.33 & tpi & L80 & 0.40 \\
L327 & $\mathbf{0 . 6 4}$ & BIO1, BIO9, tpi, slp & L250 & 0.42 \\
L332 & 0.33 & - & L263 & 0.19 \\
L334 & $\mathbf{0 . 5 9}$ & tpi & L268 & 0.28 \\
L335 & $\mathbf{0 . 5 8}$ & BIO2, tpi, slp & L304 & $\mathbf{0 . 6 2}$ \\
L344 & 0.33 & - & L337 & 0.18 \\
L345 & 0.41 & - & L356 & 0.42 \\
L346 & 0.47 & BIO4, BIO16, asp & L375 & 0.28 \\
\hline
\end{tabular}

slp, slope; tip, Topographic position index; asp, aspect

3.4. LGM, present and future distribution of $R$. aureum.

The average training AUC for ten replicate runs is 0.981, and the standard deviation is 0.035 which indicated an excellent predictive model performance. Minimum training presence logistic threshold was 0.15. The predicted distribution of $R$. aureum (Figure 4B ) is consistent with the present distribution records including Changbai Mountain, Wangtiane Mountain, Laobai Mountain and North Koera, showing that the distribution of $R$. aureum is conditioned by environmental factors. The distribution of the LGM based on CCSM4 (Figure 4A ) was substantially different from the present. The assessed distribution of $R$. aureum during the LGM was expanded to Korean Autonomous Prefecture of Yanbian and northern mountain of Korean peninsula. The predicted future and present distribution of $R$. aureum was considerably different in geographical range (Figure 4C, 4D ). The major difference was that the predicted future suitable habitats showed a significant lessening in comparison with the present one. Only the peak of Changbai Mountain and some area in the North Korea would suitable for $R$. aureum under the rcp85 scenario. Loss of suitable habitats because of the climate changing indicated a drastically range contraction. 

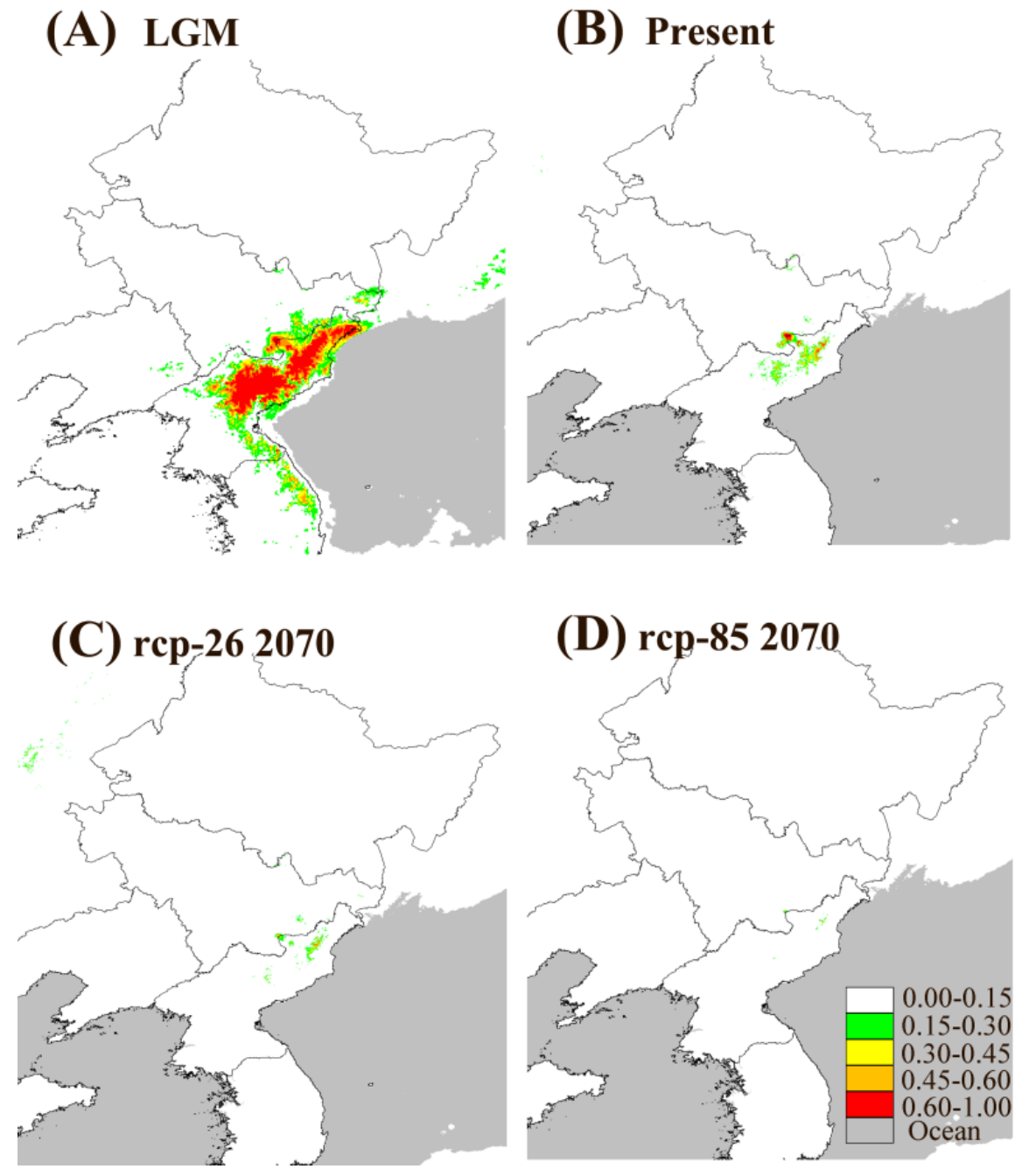

Figure 4. Potential distributions as the probability of occurrence for $R$. aureum . Suitability values indicate logistic probabilities ranging from $0-1$, with increasingly darker shades of red with increasing habitat suitability. (A) Last Glacial Maximum (LGM) scenario; (B) present scenario; (C) RCP 26 scenario; (D) RCP 85 scenario (MAXENT v3.3.3k \& Adobe Photoshop CS3).

\section{Discussion}

R. aureum exhibited a higher level of genetic diversity at the species level $(\mathrm{I}=0.584, \mathrm{H}=0.402)$ than other Ericaceae species similarly researched with AFLP markers, such as $R$. ledebourii, $R$. dauricum, $R$. sichotense (Tikhonova, Polezhaeva, \& Pimenova, 2012); and the high level of genetic diversity were in accordance with studies on other arctic and alpine species (H. A. PERSSON, 2001). High genetic diversity was also observed of $R$. aureum by RAPD and ISSR markers at the species level(Liu et al., 2012). Plant species with wide altitudinal ranges encounter different environmental conditions across the elevation gradient, which may lead to genetic variation as well as phenotypic variation among populations (Anna-Barbara Utelli, 1995; Forsman, 2014; Nicotra et al., 2015; Ohsawa \& Ide, 2008). R. aureum is a long-lived, perennial, evergreen, dwarf shrub which altitude range from $1000 \mathrm{~m}$ to $2600 \mathrm{~m}$ in alpine regions. Along elevational gradients of alpine area, large changes in environmental factors, such as temperature, precipitation (Figure $\mathbf{C}$ ), solar radiation, and wind, occur over short distances, resulting from obvious changes in the selection pressures of $R$. aureumindividuals. Heterogeneous habitats strengthen disruptive selection to increase variation and diver- 
gent selection pressures promote the evolution of traits adapted to their local environment, (Freeland, 2005). Divergent selection can promote genetic differentiation by reducing gene flow among sites with contrasting ecological conditions(Forester, Jones, \& Joost, 2016). Results also showed that the genetic variability was even greater among populations $(68.87 \%)$ but smaller within populations $(31.13 \%)$, and there are high levels of differentiation among populations $\left(\Phi_{\Sigma T}=0.689\right)$. Meanwhile, the high population differentiation could possibly accelerate local adaptation. Local adaptation and directional selection should have locus-specific effects of reducing genetic diversity within populations and increasing differentiation between populations (Magdy et al., 2016). Furthermore, long-lived perennial species with mixed breeding systems usually have relatively high genetic diversity (Nybom \& Bartish, 2000). In the long-term evolutionary process, the high genetic variation held by $R$. aureummay have provided abundant genotypes for its adaptation to changing climatic conditions. There were some populations got the relatively lower genetic diversity than others. Population N7 inhabit on the low altitude in the coniferous forest which has a forest barrier from the others. Possible explanation for the low diversity found in the population is that small populations and habitat fragmentation are more susceptible pollen limitation, limited gene flow and genetic drift leading to loss of genetic diversity (Norman C. Ellstrand 1993; Vranckx, Jacquemyn, Muys, \& Honnay, 2012).

Genetic divergence between populations is shaped by a combination of drift, migration, and selection, yielding patterns of isolation-by-distance (IBD) and isolation-by-environment (IBE)(Weber, Bradburd, Stuart, Stutz, $\&$ Bolnick, 2017). Some researches on population genetic structure discovered that IBD plays a more important role in intraspecific genetic differentiation than IBE(Mosca, González-Martinez, \& Neale, 2014), however, IBE was implied to have a stronger effect than IBD on genetic structure in other plant taxa(Gray et al., 2014). A stronger effect of IBE versus IBD was found for the genetic differentiation of $R$. aureum . A Mantel test, partial Mantel test and MMRR analysis all supported the effect of isolation by environmental distance. In the cluster analysis, the fact that some geographically close populations are separated by larger genetic divergence than expected also proved the IBD is not the major driver of population divergence of $R$. aureum . The prominence of IBE suggests factors related to the environment play a greater role in divergence of $R$. aureumpopulations than geographical isolation. $R$. aureum lives in diversified habitats across its distribution region, and ecological landscape heterogeneity may influence gene flow and connectivity among populations that are adapted to different environments. Possible mechanisms responsible for IBE are selection pressures from climate and relief factors.

In identifying outlier loci or adaptive loci, we sought to determine how selection may play a role in shaping genetic differentiation and adaptation along sharp environmental clines. All 42 outlier loci identified by both BayeScan and Dfdist was undergoing putative diversifying selection and balancing selection (Figure 3). Most of the outlier associated with environmental predictors across the alpine environmental gradient (Table 4), suggesting these regions of the genome seem to be diverging and that climate may play a role. Most outliers were associated with temperatures related predictors (especially BIO1 and BIO3), probably due to the steep gradient in temperatures along our sampled region. In addition, many outliers were associated with precipitation and relief related environmental predictors, suggesting that precipitation and relief may also be exerting spatially divergent pressure on genetic. As expected, temperature, precipitation were estimated as the major driving factors influencing allele frequencies at outlier loci, consistent with other studies examining drivers of adaptive genetic divergence in plants(Manel, Poncet, Legendre, Gugerli, \& Holderegger, 2010a; Yoder et al., 2014). Temperatures and precipitation factors are very important for plant growth, development, survival, reproduction and defense(Poncet et al., 2010). However, there are little researches has found the relief related factors influence the adaptive genetic divergence(Manel et al., 2010a). In this study, we found many outlier loci were related to the relief factors, such as 5 outlier loci were related to topographic position index (tpi), 4 outlier loci were related to aspect (asp), 2 outlier loci were related to slope (slp) with high values of $R_{\mathrm{adj}}{ }^{2}$. The relief has complex indirect effects on the combination of snow distribution and slope specific interception of radiation, and has the direct influence of exposure on microclimate during the growing season(Korner, 2003).

We used MAXENT to predict the distribution of $R$. aureum under LGM (Last Glacial Maximum), present and future climate conditions. MAXENT captured well a major portion of current distribution of $R$. aureum 
- With the climate changing from the LGM to future, $R$. aureum decreased its future distribution range under a climatic warming scenario, especially under the RCP (Representative Concentration Pathways) 85 scenario which higher level greenhouse gases are emitted than RCP 26 in the years to come. We found the suitable distribution range of $R$. aureum would be reduced to the high altitude tundra area but would lose the low altitude area in Changbai Mountain. This is consistent with previous studies on other alpine area. Ecosystems at high latitudes and altitudes are particularly sensitive to climate change. Climate change is causing many species to shift their geographical ranges as reviewed in many researches (Bellard, Bertelsmeier, Leadley, Thuiller, \& Courchamp, 2012; Dawson, Jackson, House, Prentice, \& Mace, 2011). The abundance and dominance of shrub species have increased in alpine and subarctic tundra ecosystems in recent decades (Brandt, Haynes, Kuemmerle, Waller, \& Radeloff, 2013; Myers-Smith et al., 2011; Myers-Smith et al., 2015; Sturm, Racine, \& Tape, 2001; Sturm et al., 2005; Tape, Sturm, \& Racine, 2006) and climate warming has been considered the dominant factor driving these range expansions of shrubs (Brandt et al., 2013; Li et al., 2016; Naito \& Cairns, 2011; Walker et al., 2006; Yu, Luedeling, \& Xu, 2010). As an effect of global warming, upward shifting of plant species in high mountain systems was predicted for the near future(Pauli, Gottfried, \& Grabherr, 1996). Climate-induced range shifts and population declines are expected to increase the prevalence of population bottlenecks and reduce genetic diversity within and among species. Long-lived species are particularly vulnerable to climate changes because they experience longer generation times, lower population turnover rates and slower rates of evolution(Staudinger et al., 2012).

\section{Conclusions}

In summary, by using AFLP markers, landscape genetic, and species distribution modeling analysis together, we are able to identify many environmental factors that have influenced on the genetic diversity and genetic structure, and we can predict the potential distribution area of $R$. aureum. Our analyses revealed high genetic variation and differentiation among populations and moderate levels of genetic diversity within populations of $R$. aureum. A significant correlation between genetic distance and environmental distance was identified, which suggested that environmental factors were the primary cause of the population differentiation. 42 outlier loci were identified in 36 populations of $R$. aureum alone the environmental gradient and most of the outlier loci are associated with environmental factors, suggesting that these loci are linked to genes that are involved in the adaptability of $R$. aureum to environment. The SDM indicates that climate change drastically reduces the potential distribution range of $R$. aureum. An urgent area of future study is identification of genomic regions that are associated with environment factors by RAD-Seq(Hohenlohe, Catchen, \& Cresko, 2012) and EST (expressed sequence tags). We should take measures to protect this species, such as translocate the populations or establish captive populations that would otherwise go extinct.

Author Contributions: Conceptualization, X.C; methodology, W.Z., Y.H., Y.Z. and L.L.; formal analysis, W.Z. and X.L.W.; data curation, W.Z. and J.N.L; writing — original draft preparation, W.Z.; writing - review and editing, X.C. All authors have read and agreed to the published version of the manuscript.

Funding: This work was supported by grants from the Ji Lin province Natural Science Foundation [20190201298JC].

Conflicts of Interest: The authors declare no conflict of interest.

Appendix A

Table A. The primers used for AFLP analysis

\begin{tabular}{lll}
\hline Adaptors & Adaptors & Adaptors \\
\hline & EcoRI-1 5'-CTCGTAGACTGCGTACC-3' & MseI-1 5'-GACGATGAGTCCTGAG-3' \\
& EcoRI2 5'-AATTGGTACGCAGTCTAC-3' & MseI-2 5'-TACTCAGGACTCAT-3' \\
Preamplification primers & Preamplification primers & Preamplification primers \\
& 5'-GACTGCGTACCAATTCA-3' & 5'-GATGAGTCCTGAGTAAC-3' \\
Selective amplification primers & Selective amplification primers & Selective amplification primers
\end{tabular}




\begin{tabular}{lll}
\hline Adaptors & Adaptors & Adaptors \\
\hline AFLP-1 & 5'-GACTGCGTACCAATTCACC-3' & 5'-GATGAGTCCTGAGTAACAA-3' \\
AFLP-2 & 5'-GACTGCGTACCAATTCACC-3' & 5'-GATGAGTCCTGAGTAACTA-3' \\
AFLP-3 & 5'-GACTGCGTACCAATTCAGG-3' & 5'-GATGAGTCCTGAGTAACAT-3' \\
AFLP-4 & 5'-GACTGCGTACCAATTCAGG-3' & 5'-GATGAGTCCTGAGTAACAG-3' \\
AFLP-5 & 5'-GACTGCGTACCAATTCACA-3' & 5'-GATGAGTCCTGAGTAACTA-3' \\
AFLP-6 & 5'-GACTGCGTACCAATTCACA-3' & 5'-GATGAGTCCTGAGTAACTT-3' \\
AFLP-7 & 5'-GACTGCGTACCAATTCACT-3' & 5'-GATGAGTCCTGAGTAACAA-3' \\
AFLP-8 & 5'-GACTGCGTACCAATTCACT-3' & 5'-GATGAGTCCTGAGTAACAT-3' \\
AFLP-9 & 5'-GACTGCGTACCAATTCACT-3' & 5'-GATGAGTCCTGAGTAACAC-3' \\
AFLP-10 & 5'-GACTGCGTACCAATTCACT-3' & 5'-GATGAGTCCTGAGTAACTA-3' \\
\hline
\end{tabular}

Appendix B

Table B. The distribution records of $R$. aureum

\begin{tabular}{llllll}
\hline Sites & Longitude/E & Latitude/N & Sites & Longitude/E & Latitude/N \\
\hline 1 & 137.8000 & 35.80000 & 22 & 128.0485 & 42.03802 \\
2 & 142.8667 & 43.71667 & 23 & 128.0385 & 42.02957 \\
3 & 142.8833 & 43.55000 & 24 & 128.0515 & 42.04300 \\
4 & 142.8667 & 43.55000 & 25 & 128.0001 & 41.99007 \\
5 & 142.8667 & 43.68333 & 26 & 128.0119 & 41.98912 \\
6 & 138.3667 & 36.00000 & 27 & 128.0049 & 41.98713 \\
7 & 128.0664 & 42.02920 & 28 & 128.0006 & 41.97795 \\
8 & 128.0678 & 42.04018 & 29 & 128.0636 & 41.97879 \\
9 & 128.0686 & 42.04202 & 30 & 128.0826 & 41.95675 \\
10 & 128.0706 & 42.04575 & 31 & 128.0794 & 41.95501 \\
11 & 128.0699 & 42.05552 & 32 & 128.0762 & 41.95376 \\
12 & 128.0630 & 42.05935 & 33 & 128.0752 & 41.95178 \\
13 & 128.0687 & 42.09014 & 34 & 128.0488 & 41.94115 \\
14 & 128.1910 & 42.13372 & 35 & 128.0714 & 42.05420 \\
15 & 128.0250 & 41.99727 & 36 & 128.0738 & 42.04927 \\
16 & 128.0249 & 42.00210 & 37 & 128.0749 & 42.04517 \\
17 & 128.0212 & 42.01297 & 38 & 128.0681 & 42.04015 \\
18 & 128.0252 & 42.01640 & 39 & 128.0431 & 44.10465 \\
19 & 128.0425 & 42.02153 & 40 & 128.0426 & 44.10325 \\
20 & 128.0402 & 42.02443 & 41 & 127.9020 & 41.72863 \\
21 & 128.0433 & 42.03347 & 42 & 127.9011 & 41.72845 \\
\hline
\end{tabular}

Appendix C 


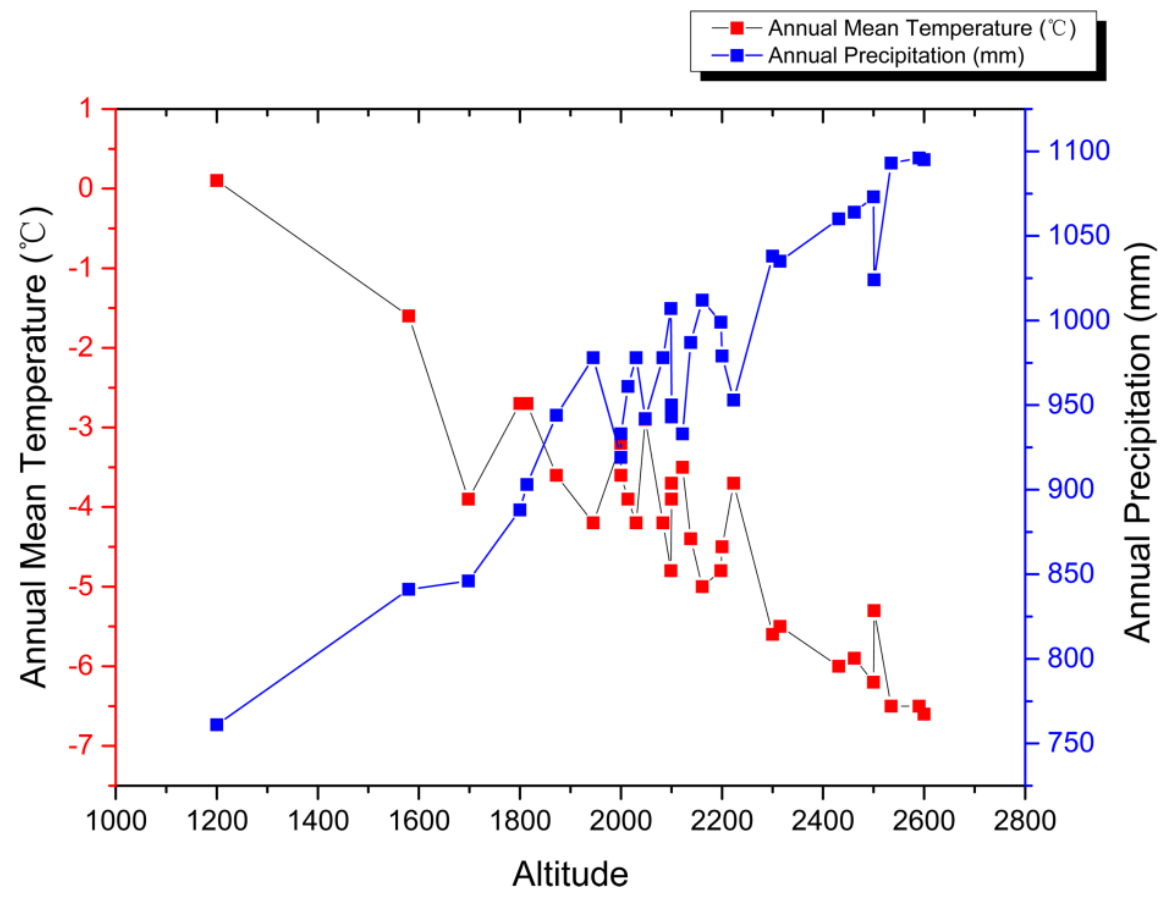

Figure C. The different annual mean temperature and annual precipitation along the altitude. The data was obtained from WordlClim database (http://worldclim.org/).

Data Accessibility Statement

We agree to deposit our data to a public repository. The data that support the findings of this study will be deposited inDryad upon acceptance.

\section{References}

And, M. D. L., \& Hamrick, J. L. (1984). Ecological Determinants of Genetic Structure in Plant Populations. Annual Review of Ecology \&3 Systematics, 15 (1), 65-95.

Anna-Barbara Utelli, W. H., Hans-Jakob Zopfi (1995). Phenotypic plasticity in alpine Erigeron species (Asteraceae). Nordic Journal of Botany, 15 (5), 483-492.

Bassam BJ, C.-A. G., Gresshoff PM (1991). Fast and sensitive silver staining of DNA in polyacrylamide gels. analytical biochemistry, $196,80-83$.

Beaumont, M. A., \& Balding, D. J. (2004). Identifying adaptive genetic divergence among populations from genome scans. Molecular Ecology, 13 (4), 969-980.

Beaumont, M. A., \& Nichols, R. A. (1996). Evaluating Loci for Use in the Genetic Analysis of Population Structure. Proceedings of the Royal Society of London, 263 (1377), 1619-1626.

Bellard, C., Bertelsmeier, C., Leadley, P., Thuiller, W., \& Courchamp, F. (2012). Impacts of climate change on the future of biodiversity.Ecol Lett, 15 (4), 365-377. doi:10.1111/j.1461-0248.2011.01736.x

Bensch, S., \& Akesson, M. (2005). Ten years of AFLP in ecology and evolution: why so few animals? Molecular Ecology, 14 (10), 2899-2914.

Bohm, R., Auer, I., Brunetti, M., Maugeri, M., Nanni, T., \& Schoner, W. (2001). Regional temperature variability in the European Alps: 1760-1998 from homogenized instrumental time series. International Journal of Climatology, 21 (14), 1779-1801. 
Bothwell, H., Bisbing, S., Therkildsen, N. O., Crawford, L., Alvarez, N., Holderegger, R., \& Manel, S. (2013). Identifying genetic signatures of selection in a non-model species, alpine gentian (Gentiana nivalis L.), using a landscape genetic approach. Conservation Genetics, 14 (2), 467-481.

Brandt, J. S., Haynes, M. A., Kuemmerle, T., Waller, D. M., \& Radeloff, V. C. (2013). Regime shift on the roof of the world: Alpine meadows converting to shrublands in the southern Himalayas. Biological Conservation, 158 , 116-127. doi:10.1016/j.biocon.2012.07.026

Breshears, D. D., Huxman, T. E., Adams, H. D., Zou, C. B., \& Davison, J. E. (2008). Vegetation synchronously leans upslope as climate warms.Proc Natl Acad Sci U S A, 105 (33), 11591-11592. doi:10.1073/pnas.0806579105

Bueno, M. L., Pennington, R. T., Dexter, K. G., Kamino, L. H. Y., Pontara, V., Neves, D. M., . . . de Oliveira, A. T. (2017). Effects of Quaternary climatic fluctuations on the distribution of Neotropical savanna tree species. Ecography, 40 (3), 403-414. doi:10.1111/ecog.01860

Byars, S. G., Papst, W., \& Hoffmann, A. A. (2007). LOCAL ADAPTATION AND COGRADIENT SELECTION IN THE ALPINE PLANT, POA HIEMATA, ALONG A NARROW ALTITUDINAL GRADIENT. Evolution, 61 (12), 2925-2941.

Chartier, M., Pélozuelo, L., Buatois, B., Bessière, J. M., \& Gibernau, M. (2013). Geographical variations of odour and pollinators, and test for local adaptation by reciprocal transplant of two European Arum species. Functional Ecology, 27 (6), 1367-1381.

Coop, G., Witonsky, D., Di Rienzo, A., \& Pritchard, J. K. (2010). Using environmental correlations to identify loci underlying local adaptation.Genetics, 185 (4), 1411-1423. doi:10.1534/genetics.110.114819

Davis, M. B., \& Shaw, R. G. (2001). Range shifts and adaptive responses to Quaternary climate change. Science, 292 (5517), 673-679. doi:10.1126/science.292.5517.673

Davis, M. B., Shaw, R. G., \& Etterson, J. R. (2008). EVOLUTIONARY RESPONSES TO CHANGING CLIMATE. ecology, 86 (7), 1704-1714.

Dawson, T. P., Jackson, S. T., House, J. I., Prentice, I. C., \& Mace, G. M. (2011). Beyond predictions: biodiversity conservation in a changing climate. Science, 332 (6025), 53-58. doi:10.1126/science.1200303

E.E.K. Donald A. Falk, E. O. G. (2001). An introduction to restoration genetics .

Earl, D. A., \& Vonholdt, B. M. (2012). STRUCTURE HARVESTER: a website and program for visualizing STRUCTURE output and implementing the Evanno method. Conservation Genetics Resources, 4 (2), 1-3.

Ehrich, D. (2006). AFLPdat: a collection of R functions for convenient handling of AFLP data. Mol. Ecol. Notes, 6 , 603-604.

Erickson, D. L., Hamrick, J. L., \& Kochert, G. D. (2004). Ecological determinants of genetic diversity in an expanding population of the shrub Myrica cerifera. Molecular Ecology, 13 (6), 1655-1664.

Evanno, G., Regnaut, S., \& Goudet, J. (2005). Detecting the number of clusters of individuals using the software STRUCTURE: a simulation study. Mol Ecol, 14 (8), 2611-2620. doi:10.1111/j.1365-294X.2005.02553.x

Excoffier, L., Hofer, T., \& Foll, M. (2009). Detecting loci under selection in a hierarchically structured population. Heredity (Edinb), 103 (4), 285-298. doi:10.1038/hdy.2009.74

Excoffier, L., \& Lischer, H. E. (2010). Arlequin suite ver 3.5: a new series of programs to perform population genetics analyses under Linux and Windows. Mol Ecol Resour, 10 (3), 564-567.

Fischer, M. C., Foll, M., Excoffier, L., \& Heckel, G. (2011). Enhanced AFLP genome scans detect local adaptation in high-altitude populations of a small rodent (Microtus arvalis). Molecular Ecology, 20 (7), $1450-1462$. 
Foll, M., \& Gaggiotti, O. (2008). A genome-scan method to identify selected loci appropriate for both dominant and codominant markers: a Bayesian perspective. Genetics, 180 (2), 977-993.

Forester, B. R., Jones, M. R., \& Joost, S. (2016). Detecting spatial genetic signatures of local adaptation in heterogeneous landscapes.25 (1), 104-120. doi:10.1111/mec.13476

Forsman, A. (2014). Rethinking phenotypic plasticity and its consequences for individuals, populations and species. Heredity (Edinb), 115 (4), 276-284. doi:10.1038/hdy.2014.92

Frankham, R. (2005). Genetics and extinction. Biological Conservation, 126 (2), 131-140. doi:10.1016/j.biocon.2005.05.002

Freeland, J. R. (2005). Molecular ecology : John Wiley \& Sons Ltd.

Gonzalez-Martinez, S. C., Krutovsky, K. V., \& Neale, D. B. (2006). Forest-tree population genomics and adaptive evolution. New Phytol, 170 (2), 227-238. doi:10.1111/j.1469-8137.2006.01686.x

Goslee, S. C., \& Urban, D. L. (2007). The ecodist Package for Dissimilarity-based Analysis of Ecological Data. Journal of Statistical Software, 22 (7), 1-19.

Gray, M. M., St Amand, P., Bello, N. M., Galliart, M. B., Knapp, M., Garrett, K. A., . . Akhunov, E. D. (2014). Ecotypes of an ecologically dominant prairie grass (Andropogon gerardii) exhibit genetic divergence across the US Midwest grasslands' environmental gradient.Molecular Ecology, 23 (24), 6011-6028.

H. A. PERSSON, B. A. G. (2001). The extent of clonality and genetic diversity in lingonberry (Vaccinium vitis-idaea L.) revealed by RAPDs and leaf-shape analysis. Molecular Ecology, 10 (6), 1385-1397.

Hancock, A. M., Brachi, B., Faure, N., Horton, M. W., Jarymowycz, L. B., Sperone, F. G., . . . Bergelson, J. (2011). Adaptation to climate across the Arabidopsis thaliana genome. Science, 334 (6052), 83-86. doi:10.1126/science.1209244

Harris, B. (1961). Theory of probability : Oxford at the Clarendon Press.

Hijmans, R. J., Cameron, S. E., Parra, J. L., Jones, P. G., \& Jarvis, A. (2005). Very high resolution interpolated climate surfaces for global land areas. International Journal of Climatology, 25 (15), 1965-1978.

Hijmans, R. J., \& Graham, C. H. (2006). The ability of climate envelope models to predict the effect of climate change on species distributions. Glob Chang Biol, 12 (12), 2272-2281.

Hijmans, R. J., Guarino, L., Cruz, M., \& Rojas, E. (2001). Computer tools for spatial analysis of plant genetic resources data: 1. DIVA-GIS.Plant Genetic Resources Newsletter, 127, 15-19.

Hohenlohe, P. A., Catchen, J., \& Cresko, W. A. (2012). Population genomic analysis of model and nonmodel organisms using sequenced RAD tags : Humana Press.

Holderegger, R., Buehler, D., Gugerli, F., \& Manel, S. (2010). Landscape genetics of plants. Trends Plant Sci, 15 (12), 675-683. doi:10.1016/j.tplants.2010.09.002

Hovenden, M. J., \& Jkvander, S. (2004). Nature vs nurture in the leaf morphology of Southern beech, Nothofagus cunninghamii (Nothofagaceae).New Phytologist, 161 (2), 585-594.

Jakobsson, M., \& Rosenberg, N. A. (2007). CLUMPP: a cluster matching and permutation program for dealing with label switching and multimodality in analysis of population structure. Bioinformatics, 23 (14), 1801-1806.

Joost, S., Bonin, A., Bruford, M. W., Despres, L., Conord, C., Erhardt, G., \& Taberlet, P. (2007). A spatial analysis method (SAM) to detect candidate loci for selection: towards a landscape genomics approach to adaptation. Mol Ecol, 16 (18), 3955-3969. doi:10.1111/j.1365-294X.2007.03442.x

Körner, C. (2003). Alpine Plant Life-Functional Plant Ecology of High Mountain Ecosystems . Berlin Heidelberg: Springer-Verlag. 
Kremen, C., Cameron, A., Moilanen, A., Phillips, S. J., Thomas, C. D., Beentje, H., . . Zjhra, M. L. (2008). Aligning conservation priorities across taxa in Madagascar with high-resolution planning tools.Science, 320 (5873), 222-226.

Kudo, G. (1992). Effect of snow-free duration on leaf life-span of four alpine plant species. Canadian Journal of Botany, 70 (8), 1684-1688.

Kudo, G. (1993). Relationships between Flowering Time and Fruit Set of the Entomophilous Alpine Shrub, Rhododendron aureum (Ericaceae), Inhabiting Snow Patches. American Journal of Botany, 80 (11), 13001304

Lenoir, J., Gegout, J. C., Marquet, P. A., de Ruffray, P., \& Brisse, H. (2008). A significant upward shift in plant species optimum elevation during the 20th century. Science, 320 (5884), 1768-1771. doi:10.1126/science.1156831

Li, B., Heijmans, M. M. P. D., Berendse, F., Blok, D., Maximov, T., \& Sass-Klaassen, U. (2016). The role of summer precipitation and summer temperature in establishment and growth of dwarf shrub Betula nana in northeast Siberian tundra. Polar Biology, 39 (7), 1245-1255. doi:10.1007/s00300-015-1847-0

Liu, Y.-F., Xing, M., Zhao, W., Fan, R.-J., Luo, S., \& Chen, X. (2012). Genetic diversity analysis of Rhododendron aureum Georgi (Ericaceae) located on Changbai Mountain using ISSR and RAPD markers. Plant Systematics and Evolution, 298 (5), 921-930. doi:10.1007/s00606-012-0601-0

Luikart, G., England, P. R., Tallmon, D., Jordan, S., \& Taberlet, P. (2003). The power and promise of population genomics: from genotyping to genome typing. Nature Reviews Genetics, 4 (12), 981-994.

Magdy, M., Werner, O., McDaniel, S. F., Goffinet, B., \& Ros, R. M. (2016). Genomic scanning using AFLP to detect loci under selection in the moss Funaria hygrometrica along a climate gradient in the Sierra Nevada Mountains, Spain. Plant Biol (Stuttg), 18 (2), 280-288. doi:10.1111/plb.12381

Manel, S., Poncet, B. N., Legendre, P., Gugerli, F., \& Holderegger, R. (2010a). Common factors drive adaptive genetic variation at different spatial scales in Arabis alpina. Molecular Ecology, 19 (17), 3824-3835. doi:10.1111/j.1365-294X.2010.04716.x

Manel, S., Poncet, B. N., Legendre, P., Gugerli, F., \& Holderegger, R. (2010b). Common factors drive adaptive genetic variation at different spatial scales in Arabis alpina. Mol Ecol, 19 (17), 3824-3835. doi:10.1111/j.1365294X.2010.04716.x

Merow, C., Smith, M. J., \& Silander, J. A. (2013). A practical guide to MaxEnt for modeling species' distributions: what it does, and why inputs and settings matter. Ecography, 36 (10), 1058-1069. doi:10.1111/j.16000587.2013.07872.x

Meyer, C. L., Vitalis, R., Saumitou-Laprade, P., \& Castric, V. (2009). Genomic pattern of adaptive divergence in Arabidopsis halleri, a model species for tolerance to heavy metal. Molecular Ecology, 18 (9), 2050-2062.

Mosca, E., González-Martinez, S. C., \& Neale, D. B. (2014). Environmental versus geographical determinants of genetic structure in two subalpine conifers. New Phytologist, 201 (1), 180-192.

Myers-Smith, I. H., Forbes, B. C., Wilmking, M., Hallinger, M., Lantz, T., Blok, D., . . Hik, D. S. (2011). Shrub expansion in tundra ecosystems: dynamics, impacts and research priorities.Environmental Research Letters, 6 (4), 045509. doi:10.1088/1748-9326/6/4/045509

Myers-Smith, I. H., Hallinger, M., Blok, D., Sass-Klaassen, U., Rayback, S. A., Weijers, S., . . . Wilmking, M. (2015). Methods for measuring arctic and alpine shrub growth: A review. Earth-Science Reviews, 140 , 1-13. doi:10.1016/j.earscirev.2014.10.004

Naito, A. T., \& Cairns, D. M. (2011). Patterns and processes of global shrub expansion. Progress in Physical Geography, 35 (4), 423-442. doi:10.1177/0309133311403538 
Nei, M. (1973). Analysis of gene diversity in subdivided populations.pnas, 70 , 3321-3323.

Nei, M. (1978). ESTIMATION OF AVERAGE HETEROZYGOSITY AND GENETIC DISTANCE FROM A SMALL NUMBER OF INDIVIDUALS. Genetics, 89 (3), 583-590.

Nicotra, A. B., Segal, D. L., Hoyle, G. L., Schrey, A. W., Verhoeven, K. J., \& Richards, C. L. (2015). Adaptive plasticity and epigenetic variation in response to warming in an Alpine plant. Ecol Evol, 5 (3), 634-647. doi:10.1002/ece3.1329

Norman C. Ellstrand , D. R. E. (1993). Population Genetic Consequences of Small Population Size: Implications for Plant Conservation.Annual Review of Ecology and Systematics, 24, 217-242.

Nosil, P., \& Rundle, H. D. (2012). Ecological speciation : Oxford University Press.

Nunes, V. L., Beaumont, M. A., Butlin, R. K., \& Paulo, O. S. (2011). Multiple approaches to detect outliers in a genome scan for selection in ocellated lizards (Lacerta lepida) along an environmental gradient. Mol Ecol, 20 (2), 193-205. doi:10.1111/j.1365-294X.2010.04936.x

Nybom, H., \& Bartish, I. V. (2000). Effects of life history traits and sampling strategies on genetic diversity estimates obtained with RAPD markers in plants. Perspectives in Plant Ecology, Evolution and Systematics, 3 (2), 93-114. doi:10.1078/1433-8319-00006

Ohsawa, T., \& Ide, Y. (2008). Global patterns of genetic variation in plant species along vertical and horizontal gradients on mountains. Global Ecology and Biogeography, 17 (2), 152-163. doi:10.1111/j.14668238.2007.00357.x

Parmesan, C. (2006). Ecological and Evolutionary Responses to Recent Climate Change. Annual Review of Ecology, Evolution, and Systematics, 37 (1), 637-669. doi:10.1146/annurev.ecolsys.37.091305.110100

Parmesan, C. (2006). Ecological and Evolutionary Responses to Recent Climate Change. Annual Review of Ecology Evolution 83 Systematics, 37 (1), 637-669.

Pauli, H., Gottfried, M., \& Grabherr, G. (1996). Effects of climate change on mountain ecosystems - Upward shifting of alpine plants.Supcon International Chicago IL, 8 (3).

Phillips SJ, A. R., Schapire RE. ( 2006). Maximum entropy modeling of species geographic distributions. Ecol Model, 190 (231-259).

Phillips, S. J., \& Dudik, M. (2008). Modeling of species distributions with Maxent: new extensions and a comprehensive evaluation.Ecography, 31 (2), 161-175.

Pieter Vos, R. H., Marjo Bleeker, Martin Reijans, Theo van de Lee, Miranda Hornes, Adrie Friters, Jerina Pot, Johan Paleman, Martin Kuiper and Marc Zabeau (1995). AFLP: a new technique for DNA fingerprinting.Nucleic Acids Res, 23 (21), 4407-4414.

Poncet, B. N., Herrmann, D., Gugerli, F., Taberlet, P., Holderegger, R., Gielly, L., . . . Manel, S. (2010). Tracking genes of ecological relevance using a genome scan in two independent regional population samples of Arabis alpina. Mol Ecol, 19 (14), 2896-2907. doi:10.1111/j.1365-294X.2010.04696.x

Pritchard, J. K., Stephens, M., \& Donnelly, P. (2000). Inference of population structure using multilocus genotype data. Genetics, 7 (4), 574-578.

Rana, S. K., Rana, H. K., Ghimire, S. K., Shrestha, K. K., \& Ranjitkar, S. (2017). Predicting the impact of climate change on the distribution of two threatened Himalayan medicinal plants of Liliaceae in Nepal.Journal of Mountain Science, 14 (3), 558-570. doi:10.1007/s11629-015-3822-1

RC, L. ( 1972). The apportionment of human diversity. Evol Biol, 6 , 388-398.

Rohlf, F. J. (1997). NTSYS-pc Numerical taxonomy and multivariance analysis system, version 2.02e. Exeter Software, Setauket, New York. 
Sacks, B. N., Brown, S. K., \& Ernest, H. B. (2004). Population structure of California coyotes corresponds to habitat-specific breaks and illuminates species history. Molecular Ecology, 13 (5), 1265-1275.

Savolainen, O., Pyhajarvi, T., \& Knurr, T. (2007). Gene Flow and Local Adaptation in Trees. Annual Review of Ecology, Evolution, and Systematics, 38 (1), 595-619. doi:10.1146/annurev.ecolsys.38.091206.095646

Shryock, D. F., Havrilla, C. A., DeFalco, L. A., Esque, T. C., Custer, N. A., \& Wood, T. E. (2015). Landscape genomics of Sphaeralcea ambigua in the Mojave Desert: a multivariate, spatially-explicit approach to guide ecological restoration. Conservation Genetics, 16 (6), 1303-1317.

Staudinger, M. D., Grimm, N. B., Staudt, A., Carter, S. L., Chapin Iii, F. S., Kareiva, P., . . . Stein, B. A. (Eds.). (2012) Center for Integrated Data Analytics Wisconsin Science Center.

Storz, J. F. (2005). Using genome scans of DNA polymorphism to infer adaptive population divergence. Molecular Ecology, 14 (3), 671-688.

Sturm, M., Racine, C. H., \& Tape, K. D. (2001). Increasing Shrub Abundance in the Arctic. Nature, 411 (6837), 546-547.

Sturm, M., Schimel, J., Michaelson, G., Welker, J. M., Oberbauer, S. F., Liston, G. E., . . . Romanovsky, V. E. (2005). Winter Biological Processes Could Help Convert Arctic Tundra to Shrubland.Bioscience, 55 (1), $17-26$.

Tanksley, S. D. (1993). Mapping polygenes. Annu Rev Genet, 27 , 205-233. doi:10.1146/annurev.ge.27.120193.001225

Tape, K., Sturm, M., \& Racine, C. (2006). The evidence for shrub expansion in Northern Alaska and the Pan-Arctic. Glob Chang Biol, 12 (12), 686-702.

Thuiller, W., Lavorel, S., Araujo, M. B., Sykes, M. T., \& Prentice, I. C. (2005). Climate change threats to plant diversity in Europe.Proc Natl Acad Sci U S A, 102 (23), 8245-8250. doi:10.1073/pnas.0409902102

Tikhonova, N. A., Polezhaeva, M. A., \& Pimenova, E. A. (2012). AFLP analysis of the genetic diversity of closely related rhododendron species of the section rhodorastra (Ericaceae) from Siberia and the Far East of Russia. Russian Journal of Genetics, 48 (10), 985-992.

Vranckx, G., Jacquemyn, H., Muys, B., \& Honnay, O. (2012). Meta-analysis of susceptibility of woody plants to loss of genetic diversity through habitat fragmentation. Conserv Biol, 26 (2), 228-237. doi:10.1111/j.15231739.2011.01778.x

Walker, M. D., Wahren, C. H., Hollister, R. D., Henry, G. H. R., Ahlquist, L. E., Alatalo, J. M., . . . Carroll, A. B. (2006). Plant community responses to experimental warming across the tundra biome.Proc Natl Acad Sci U S A, 103 (5), 1342-1346.

Wan, J. Z., Wang, C. J., Tan, J. F., \& Yu, F. H. (2017). Climatic niche divergence and habitat suitability of eight alien invasive weeds in China under climate change. Ecol Evol, 7 (5), 1541-1552. doi:10.1002/ece3.2684

Wang, I. J. (2013). Examining the Full Effects of Landscape Heterogeneity on Spatial Genetic Variation: A Multiple Matrix Regression Approach for Quantifying Geographic and Ecological Isolation.Evolution, 67 (12), 3403-3411.

Wang, I. J., \& Bradburd, G. S. (2014). Isolation by environment.Molecular Ecology, 23 (23), 5649-5662.

Wang, T., Wang, Z., Xia, F., \& Su, Y. (2016). Local adaptation to temperature and precipitation in naturally fragmented populations of Cephalotaxus oliveri, an endangered conifer endemic to China. Sci Rep, 6 , 25031. doi:10.1038/srep25031

Warren, D. L., \& Seifert, S. N. (2011). Ecological niche modeling in Maxent: the importance of model complexity and the performance of model selection criteria. Ecological Applications, 21 (2), 335-342. 
Weber, J. N., Bradburd, G. S., Stuart, Y. E., Stutz, W. E., \& Bolnick, D. I. (2017). Partitioning the effects of isolation by distance, environment, and physical barriers on genomic divergence between parapatric threespine stickleback. Evolution, 71 (2), 342-356. doi:10.1111/evo.13110

Wu, Z., Yu, D., Wang, Z., Li, X., \& Xu, X. (2015). Great influence of geographic isolation on the genetic differentiation of Myriophyllum spicatum under a steep environmental gradient. Sci Rep, 5 (4), 15618.

Yang, A. H., Wei, N., Fritsch, P. W., \& Yao, X. H. (2016a). AFLP Genome Scanning Reveals Divergent Selection in Natural Populations of Liriodendron chinense (Magnoliaceae) along a Latitudinal Transect.Front Plant Sci, 7 .

Yang, A. H., Wei, N., Fritsch, P. W., \& Yao, X. H. (2016b). AFLP Genome Scanning Reveals Divergent Selection in Natural Populations of Liriodendron chinense (Magnoliaceae) along a Latitudinal Transect.Front Plant Sci, 7 , 698. doi:10.3389/fpls.2016.00698

Yang, X., \& Wu, G. (1998). The strategy for conservation and sustainable utilization of biodiversity in Changbaishan Biosphere Reserve. Journal of Forestry Research, 9 (3), 217-222.

Yeh F, Y. R., Boyle T (1997). POPGENE, the user friendly shareware for population genetic analysis. Molecular Biology and Biotechnology Center, University of Alberta, Edmonton, Canada. Molecular Biology and Biotechnology Center, University of Alberta, Edmonton, Canada.

Yoder, J. B., Stanton-Geddes, J., Zhou, P., Briskine, R., Young, N. D., \& Tiffin, P. (2014). Genomic Signature of Adaptation to Climate in Medicago truncatula. Genetics, 196 (4), 1263-+.

Yu, H., Luedeling, E., \& Xu, J. (2010). Winter and spring warming result in delayed spring phenology on the Tibetan Plateau. Proc Natl Acad Sci U S A, 107 (51), 22151-22156.

Zulliger, D., Schnyder, E., \& Gugerli, F. (2013). Are adaptive loci transferable across genomes of related species? Outlier and environmental association analyses in Alpine Brassicaceae species.Molecular Ecology, 22 (6), 1626-1639.
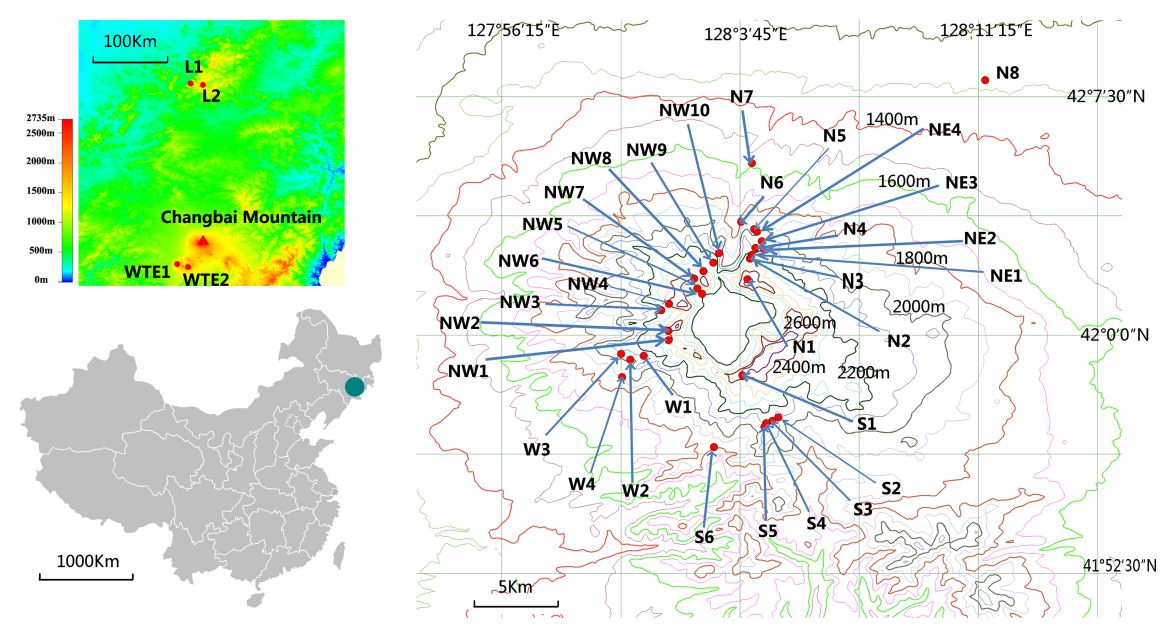

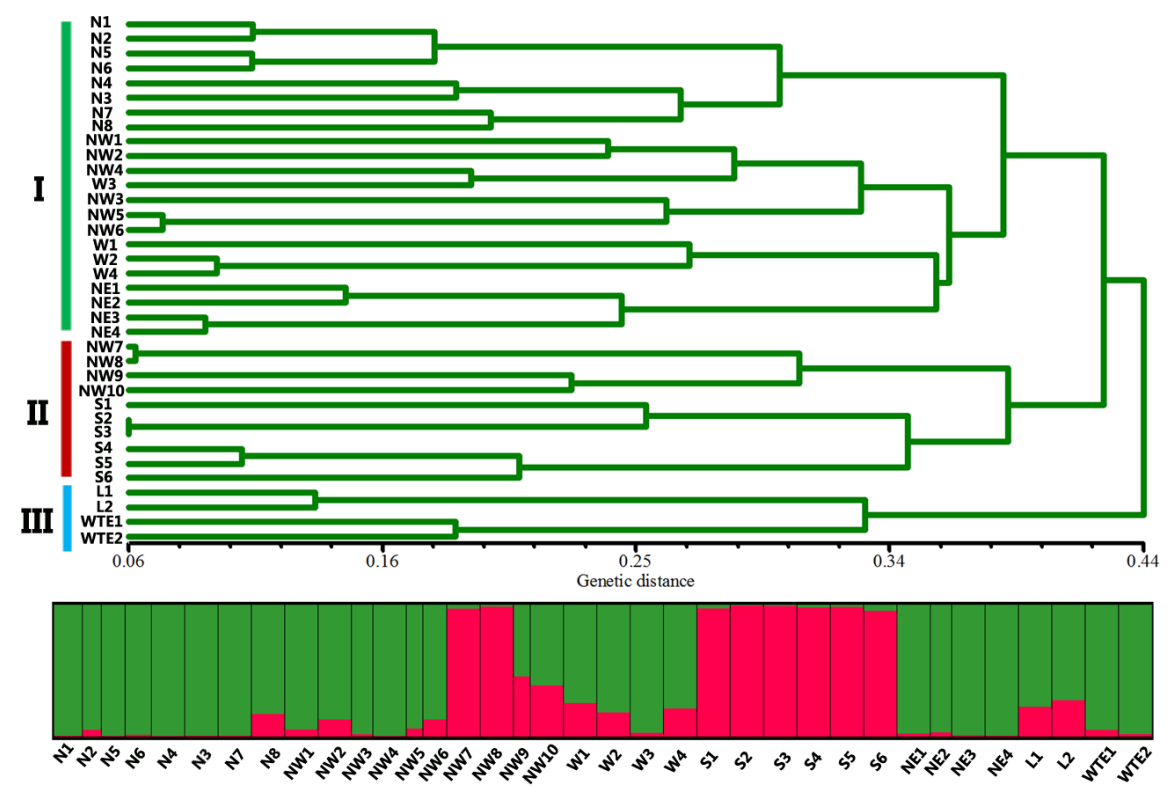


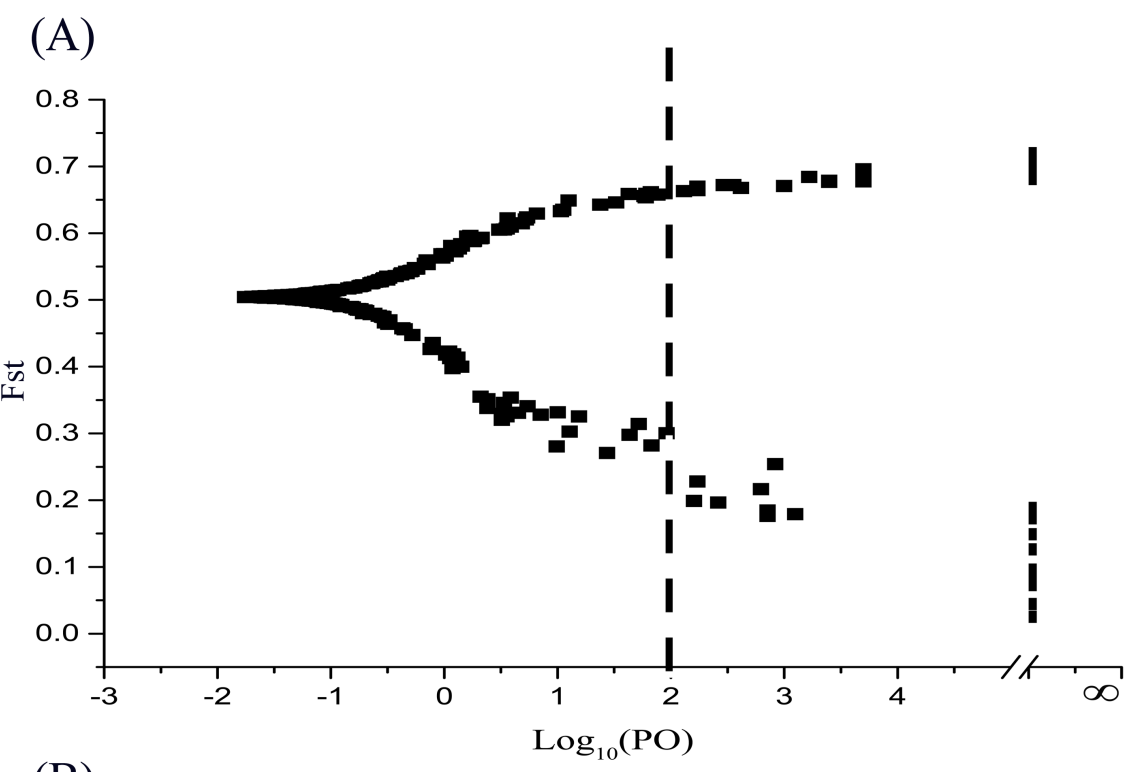

(B)

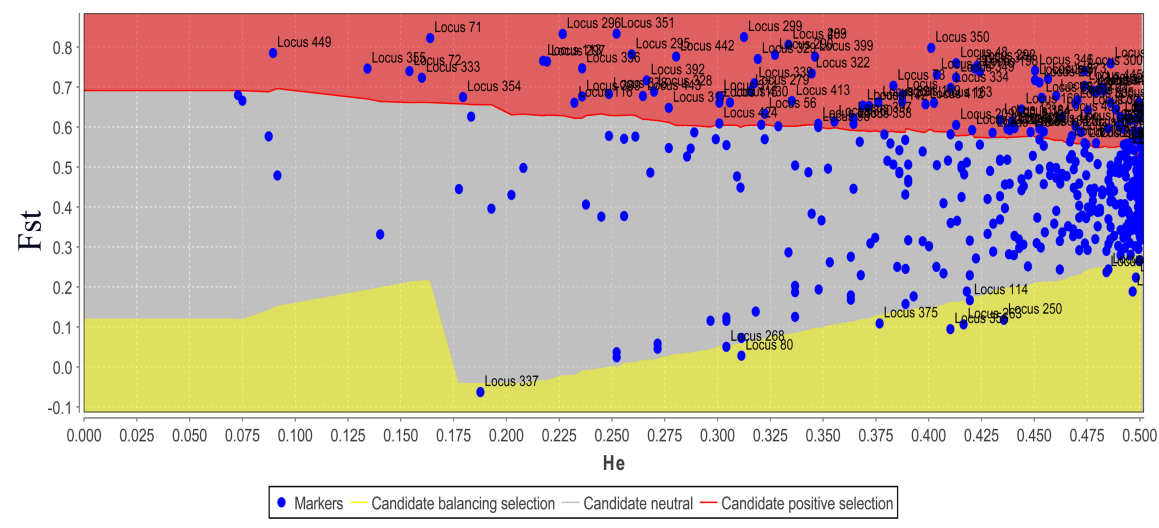



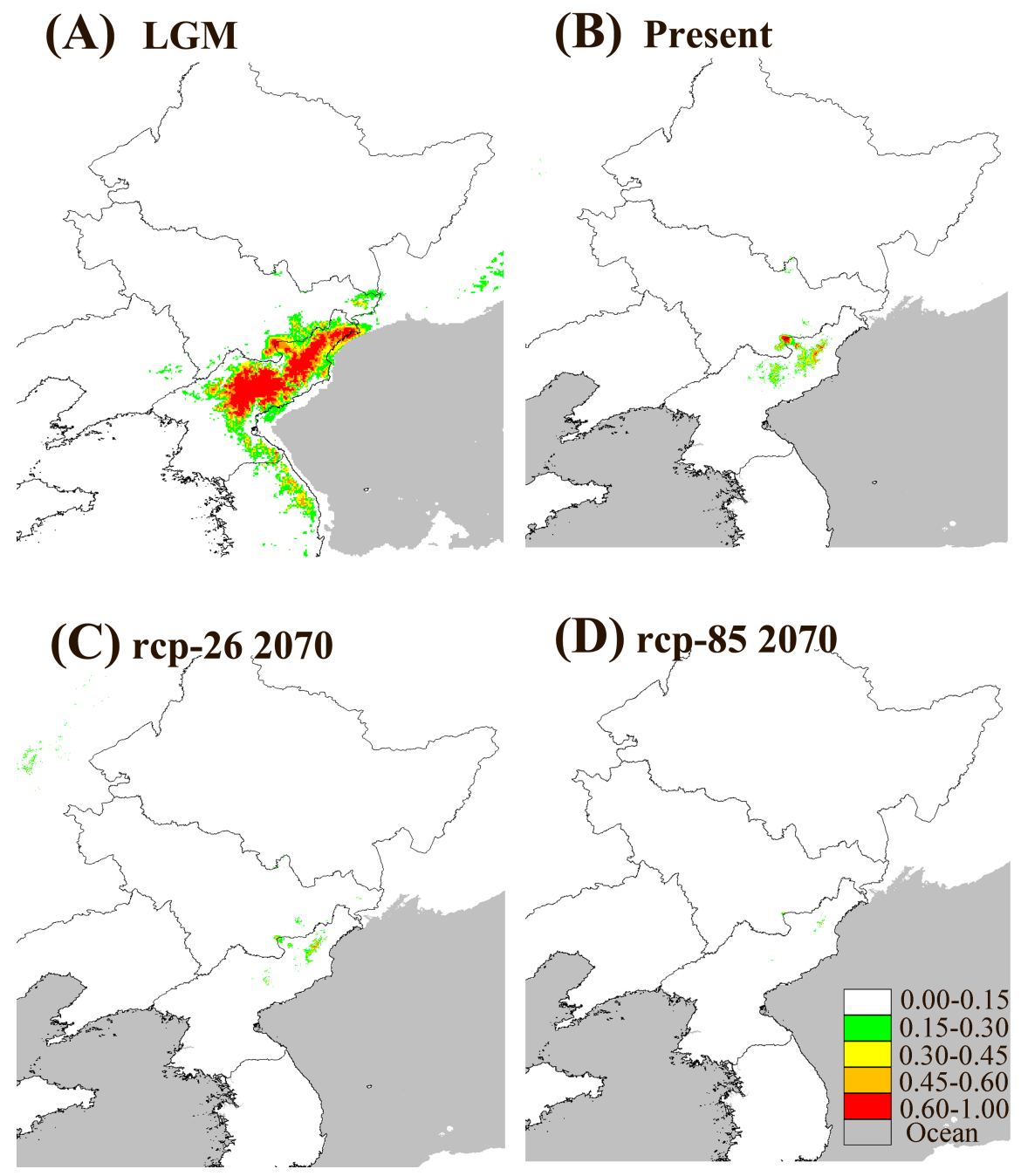